\title{
WEAKLY CONNECTED QUASI-PERIODIC OSCILLATORS, FM INTERACTIONS, AND MULTIPLEXING IN THE BRAIN*
}

\author{
EUGENE M. IZHIKEVICH ${ }^{\dagger}$
}

\begin{abstract}
We prove that weakly connected networks of quasi-periodic (multifrequency) oscillators can be transformed into a phase model by a continuous change of variables. The phase model has the same form as the one for periodic oscillators with the exception that each phase variable is a vector. When the oscillators have mutually nonresonant frequency (rotation) vectors, the phase model uncouples. This implies that such oscillators do not interact even though there might be physical connections between them. When the frequency vectors have mutual low-order resonances, the oscillators interact via phase deviations. This mechanism resembles that of the FM radio, with a shared feature-multiplexing of signals. Possible applications to neuroscience are discussed.
\end{abstract}

Key words. weakly connected neural networks, invariant manifolds, quasi-periodic oscillators, chaos, phase model, resonances, FM interactions, multiplexing, oscillatory neurocomputer, thalamocortical system

AMS subject classifications. 92B20, 34C, 34D, 58F, 82C32, 92-02, 92C20

\section{PII. S0036139997330623}

1. Introduction. Rhythms are ubiquitous in nature [47]. Recent evidence of stimulus-dependent oscillatory activity in the visual cortex suggests that rhythms may be relevant to the processing of information by the brain (see reviews $[13,40]$ ). Since the mechanism of brain rhythmic activity is far from being understood [12], we do not have a biophysically accurate model that describes completely the brain's oscillatory behavior. Fortunately, we do not need to know such a model in some special cases. For example, if we consider a weakly connected network of cortical oscillators satisfying a few technical conditions, then we can prove that any such network can be transformed into a phase model by a continuous noninvertible change of variables (Theorem 9.1 in [19]). The latter is referred to as being a canonical model due to its universality; see definitions in Appendix A. Therefore, the question of biological plausibility of the canonical model is replaced by the question of plausibility of the conditions leading to it.

In this paper we generalize the phase model theory to include multifrequency rhythmic activity. Our major assumptions are the following:

- Brain units (e.g., neurons, cortical columns, or neuronal modules) are weakly connected, as we describe in section 1.1.

- The units are autonomous quasi-periodic oscillators, as we describe in sections $1.2-1.4$.

In section 2 we prove that any weakly connected networks of quasi-periodic oscillators of the form

$$
\dot{X}_{i}=F_{i}\left(X_{i}\right)+\varepsilon G_{i}\left(X_{1}, \ldots, X_{n}, \varepsilon\right), \quad X_{i} \in \mathbb{R}^{m}, \quad i=1, \ldots, n, \quad \varepsilon \ll 1,
$$

can be transformed into a phase (canonical) model

$$
\dot{\theta}_{i}=\Omega_{i}+\varepsilon h_{i}\left(\theta_{1}, \ldots, \theta_{n}, \varepsilon\right), \quad i=1, \ldots, n,
$$

\footnotetext{
${ }^{*}$ Received by the editors November 26, 1997; accepted for publication (in revised form) October 1, 1998; published electronically October 14, 1999.

http://www.siam.org/journals/siap/59-6/33062.html

${ }^{\dagger}$ Center for Systems Science and Engineering, Arizona State University, Tempe, AZ 85287-7606 (Eugene.Izhikevich@asu.edu).
} 


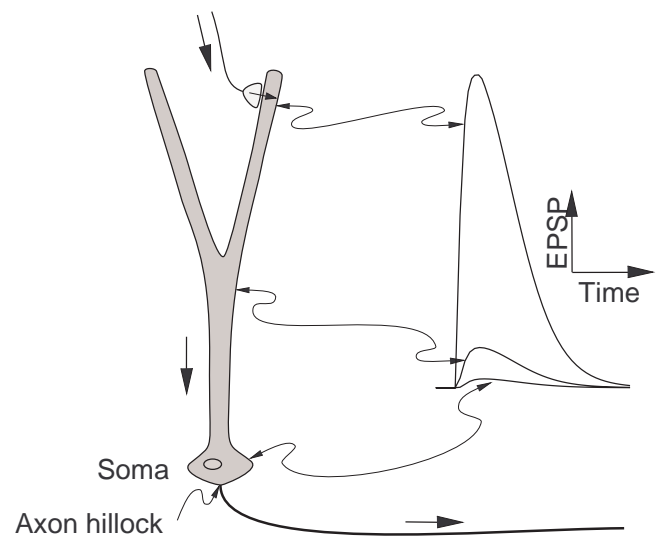

FIG. 1.1. EPSP in three different locations. The EPSP size at the soma is much smaller than the one in the vicinity of the synapse due to electrotonic attenuation. All EPSPs are simulations of nerve cable equations and are presented here for illustrational purposes only (from [19]).

by a continuous, possibly noninvertible change of variables. Here each $\theta_{i}$ is a vector of phases (angles), and $\Omega_{i}$ is a vector of frequencies of the $i$ th oscillator $X_{i}$. In section 3 we show how the phase model can be simplified further depending on the existence of resonances between the frequency vectors $\Omega_{1}, \ldots, \Omega_{n}$. We show in particular that if the frequency vectors do not have resonances, then the phase model is uncoupled even though the original weakly connected system may not be; see Theorem 3.1 for the precise statement of the result. Therefore whether or not the oscillators interact depends not only on the existence of connections between them, but also on their frequencies. We refer to such interactions as being frequency modulated (FM) interactions and discuss their possible implications in brain dynamics in section 4. In section 5 we use the well-known Wilson-Cowan and integrate-and-fire models to illustrate a few major results proven in this paper. Technical proofs and some necessary background information are presented in Appendix B.

1.1. Weakly connected systems. Weakly connected systems arise in many areas of science and engineering. Our presentation is in terms of mathematical neuroscience, and this exposition is based on section 1.3 of the book by Hoppensteadt and Izhikevich [19].

A reasonable way to characterize weakness of synaptic connections between single neurons is to consider amplitudes of postsynaptic potentials (PSPs). The PSP amplitudes may differ substantially along dendrites due to the electrotonic attenuation; see the illustration in Figure 1.1. A somatic PSP is the weakest, but it best characterizes the magnitude of the postsynaptic neuron response because the soma is near the axon hillock - the place of initiation of the action potential.

The average soma PSP is smaller than $1 \mathrm{mV}$, while the action potential emanating from the hillock region is approximately $100 \mathrm{mV}$ in amplitude. For example, PSPs in hippocampal granule cells are as small as $0.1 \pm 0.03 \mathrm{mV}$ [29], which is extremely weak compared to $100 \mathrm{mV}$ and to the mean excitatory PSP size necessary to discharge a hippocampus cell, which is $24 \pm 9 \mathrm{mV}$. Firing of a hippocampal CA3 pyramidal cell elicits EPSPs in other CA3 pyramidal cells ranging from 0.6 to $1.3 \mathrm{mV}$ [30]. Firing of the same CA3 cell can evoke EPSPs in CA1 pyramidal cells (via Schaffer collateral) 
of amplitude $0.13 \mathrm{mV}$ [38]. The majority of PSPs in pyramidal neurons of the rat visual cortex are less than $0.5 \mathrm{mV}$ in amplitude, with a range of $0.05-2.08 \mathrm{mV}$ [28]. As Mason, Nicoll, and Stratford [28] point out in their discussion section, there is an underestimate of the true range because PSPs smaller than $0.03 \mathrm{mV}$ usually go undetected.

Weak connections between individual neurons do not imply weak connections between neuronal modules consisting of these neurons, such as cortical columns. Indeed, small amplitudes of PSPs indicate that there must be many (a few hundred) presynaptic neurons firing simultaneously to make a given cell fire. However, a cortical column consists of many hundreds of neurons [33, 2]. If all those neurons fire simultaneously or within a certain time window, they may illicit an immediate and reliable response in each target cell in another cortical column, which may lead to strong connections between the columns. Such synchronous firing is not rare in the brain. For example, it occurs during stimulus-dependent oscillations in the cat visual cortex [13, 14], which implies that cortical columns in the visual cortex might not be weakly connected. In contrast, rhythmic activity in the rat hippocampus [8], in the inferotemporal cortex of the macaque, and in areas V1, MT $[4,42,48]$ involves activity of just a few cells with low firing rates. This may lead to weak connections between cortical columns in those areas.

1.2. Autonomous rhythmic activity. In this paper we study weakly connected networks that model cortical columns exhibiting autonomous rhythmic activity. Thus, we assume that rhythmic behavior of a cortical column is an endogenous property, and it is not induced by a rhythmic input from the other columns or subcortical structures (the column may require a nearly tonic excitation to maintain an appropriate level of arousal). Since the origin of brain rhythmic activity is not understood [12], it is not clear whether or when this assumption is satisfied. Answering this question may require new in vivo experiments as well as simulations of biophysically detailed neural models.

1.3. Periodic oscillators. There is a distinction between periodic and rhythmic behavior of a signal $X(t)$. The former is a mathematical notion implying that there is a period $T>0$ such that $X(t+T)=X(t)$ for all $t$, while the latter is an informal notion allowing $X(t)$ to be quasi periodic, chaotic, or noisy. Thus, the assumption that biological systems exhibit periodic activity is a drastic simplification, which is needed as a first step toward understanding such systems.

Much success is achieved when this assumption is combined with the assumption of weak connections. Indeed, it has been shown $[9,24,10]$ that behavior of a pair of weakly coupled oscillators having identical frequencies,

$$
\begin{aligned}
& \dot{X}_{1}=F_{1}\left(X_{1}\right)+\varepsilon G_{1}\left(X_{1}, X_{2}, \varepsilon\right), \\
& \dot{X}_{2}=F_{2}\left(X_{1}\right)+\varepsilon G_{2}\left(X_{1}, X_{2}, \varepsilon\right),
\end{aligned}
$$

can be described by a phase model of the form

$$
\begin{aligned}
& \varphi_{1}^{\prime}=h_{1}\left(\varphi_{2}-\varphi_{1}\right)+\mathcal{O}(\varepsilon), \\
& \varphi_{2}^{\prime}=h_{2}\left(\varphi_{1}-\varphi_{2}\right)+\mathcal{O}(\varepsilon),
\end{aligned}
$$

where each $\varphi_{i} \in \mathbb{S}^{1}$ is the phase of the $i$ th oscillator, ${ }^{\prime}=d / d \tau$, and $\tau=\varepsilon t$ is the slow time. Moreover, it has been proven [19] that there is a continuous change of variables that transforms weakly connected oscillators into their phase models. Introduction 
of the phase difference variable $\chi=\varphi_{2}-\varphi_{1}$ transforms the phase model into the form $\dot{\chi}=h_{2}(-\chi)-h_{1}(\chi)$, which can be analyzed easily. In particular, one can study stability of the in-phase $(\chi=0)$ and antiphase $(\chi=\pi)$ synchronized solutions, and other mode-locking phenomena. Studying phase models reveals many interesting facts about the dynamics of chains of weakly connected oscillators; see reviews in [35, 22].

Weakly connected oscillators having distinct frequencies have not received much attention. An exception is Ermentrout's paper [9], which considers oscillators having commensurable frequencies. Hoppensteadt and Izhikevich [19] considered oscillators having arbitrary frequencies and showed that such oscillators may interact using FM radio principles: The frequency defines the channel of communication, and the phase, which is frequency modulation, defines the information to be transmitted. That is, if the frequency is chosen inappropriately, then the oscillators do not interact even though they are interconnected. In terms of weakly connected oscillatory neurons this would mean that neurons having inappropriate mean firing rates do not communicate. If the firing rates are chosen appropriately, then they communicate via timings of spikes.

In the present paper we extend this result to include oscillators having more complicated rhythmic activity.

1.4. Quasi-periodic oscillators. EEG recordings of brain rhythmic activity rarely show periodic oscillations, even during epileptic seizures [33]. Fourier and power spectrum analysis reveal that rhythmic activity of a local field potential is "random" with a few pronounced frequencies. The most prominent are gamma $(30-100 \mathrm{~Hz})$ oscillations in the cortex and theta $(4-8 \mathrm{~Hz})$ oscillations in the hippocampus. Thus, the next natural step in modeling the brain using the oscillatory network approach is to assume that each oscillator can exhibit multifrequency oscillation, which in the simplest case may be just a collection of periodic oscillators with different frequencies (as we illustrate in section 5.3).

When the number of incommensurable frequencies is finite, the oscillator is said to be quasi periodic; see examples in Figures 1.2 and 5.2. The signals might look chaotic or noisy, but they are not. Their power spectra are discrete with peaks corresponding to the composed frequencies and their linear combinations. Possible mechanisms leading to nondiscrete spectra are discussed in section 6.6.

Let us provide some definitions. We say that a continuous rhythmic signal $X(t)$ is quasi periodic if there is a continuous function $q\left(\theta_{1}, \ldots, \theta_{k}\right)$, which is $2 \pi$-periodic in each argument, such that

$$
X(t)=q\left(\omega_{1} t, \ldots, \omega_{k} t\right) \quad \text { for all } t \geq 0,
$$

where $\Omega=\left(\omega_{1}, \ldots, \omega_{k}\right)^{\top} \in \mathbb{R}^{k}$ is a frequency vector. Let $\mathbb{T}^{k}$ denote the $k$-torus, and let $\theta=\left(\theta_{1}, \ldots, \theta_{k}\right) \in \mathbb{T}^{k}$ be the phase variable on the torus; then we may rewrite the equation above in the form

$$
X(t)=q(\theta(t)), \quad \dot{\theta}=\Omega,
$$

which is a convenient way to represent quasi-periodic oscillators. An interested reader may consult review books $[5,7,37]$ to find more about quasi-periodic oscillations and their bifurcations.

1.5. Resonant relations. We define resonant relations as in [19]. A vector of frequencies $\Omega=\left(\omega_{1}, \ldots, \omega_{n}\right)^{\top} \in \mathbb{R}^{n}$ is said to have a resonant relation if

$$
k \cdot \Omega=k_{1} \omega_{1}+\cdots+k_{n} \omega_{n}=0
$$



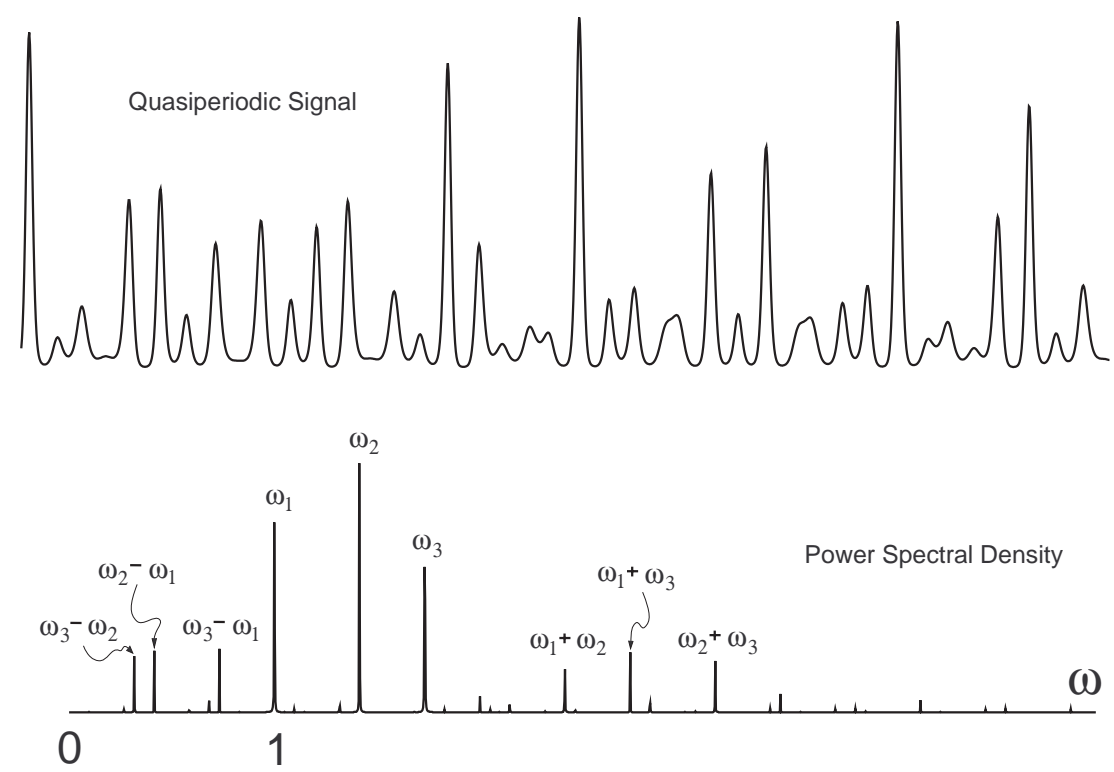

FIG. 1.2. Top: An example of a quasi-periodic signal $X(t)=\exp \left(\sin \omega_{1} t+\sin \omega_{2} t+\sin \omega_{3} t\right)$ with the frequency vector $\Omega=\left(\omega_{1}, \omega_{2}, \omega_{3}\right)=(1, \sqrt{2}, \sqrt{3})$. Bottom: Its power spectrum is always discrete.

for some nonzero integer row vector $k=\left(k_{1}, \ldots, k_{n}\right) \in \mathbb{Z}^{n}$. The sum $|k|=\sum\left|k_{i}\right|$ is the order of resonance. If $n=2$, then $\Omega=\left(\omega_{1}, \omega_{2}\right)^{\top} \in \mathbb{R}^{2}$ is resonant if and only if $\omega_{1}$ and $\omega_{2}$ are commensurable; that is, $\omega_{1} / \omega_{2}$ is a rational number.

The number of resonant relations is infinite, since they form a subgroup, $\Gamma$, of $\mathbb{Z}^{n}$. The dimension of the subgroup is at most $n-1$; therefore it has at most $n-1$ generators. It is convenient to consider them as rows of some matrix $K$, which we refer to as being a resonant matrix for $\Omega$. It is easy to check that such a matrix has the following properties:

- $K \Omega=0$.

- Each row of $K$ consists of relatively prime integers.

- Rows of $K$ are linearly independent.

Any other resonant matrix $K_{1}$ can be written in the form $K_{1}=A K$ for some matrix $A$ having integer elements; see [19].

For example, the frequency vector $\Omega=(1,2, \sqrt{2}, 2 \sqrt{2})^{\top} \in \mathbb{R}^{4}$ has a resonant matrix

$$
K=\left(\begin{array}{cccc}
-2 & 1 & 0 & 0 \\
0 & 0 & -2 & 1
\end{array}\right)
$$

The resonant subgroup here is $\Gamma=\left\{(-2 m, m,-2 k, k) \subset \mathbb{Z}^{4} \mid m, k \in \mathbb{Z}\right\}$. Notice that the rows of $K$ generate all of $\Gamma$. The matrix

$$
K^{\prime}=\left(\begin{array}{cccc}
-2 & 1 & 0 & 0 \\
-2 & 1 & -4 & 2
\end{array}\right)
$$

has linearly independent rows of relatively prime integers, and $K^{\prime} \Omega=0$. But it is 
not a resonant matrix, since the rows are not generators of $\Gamma$ (none of their linear combinations with integer coefficients produce the vector $(0,0,-2,1))$.

Without loss of generality we may assume that $\Omega$ in (1.1) is nonresonant. Indeed, if it has resonances, then the number of independent arguments of $q$ may be reduced. In particular, if $\Omega$ is proportional to a vector of integers, then $q$ may have only one argument, and hence $X(t)$ is periodic in this case.

1.5.1. Chaotic oscillators. One should be warned that going from periodic to quasi-periodic oscillations is a giant step that cannot be summarized by mere addition of another dimension. Indeed, many interesting nonlinear phenomena can occur in quasi-periodic oscillators that cannot occur in periodic ones. For example, arbitrary small perturbations of a quasi-periodic oscillator,

$$
\dot{\theta}=\Omega+\epsilon h(\theta), \quad \epsilon \ll 1
$$

may produce strange attractors and toroidal chaos $[36,32,3]$ when $k \geq 3$. This is not possible for periodic oscillators, i.e., when $k=1$.

In this paper we study weakly connected networks of quasi-periodic oscillators. Since any such oscillator is $\epsilon$-close to being chaotic, our results can be extended for weakly connected networks of such chaotic oscillators provided that the size of perturbation $\epsilon$ has the same order as the strength of connections $\varepsilon$; see equation (2.1) below. That is, we incorporate $\epsilon$-perturbations into the connection function $\varepsilon G_{i}$.

2. Derivation of the phase model. We implicitly assume that all manifolds and functions arising here are as smooth as necessary for our manipulations.

THEOREM 2.1 (phase model for weakly connected quasi-periodic oscillators). Consider a weakly connected system of the form

$$
\dot{X}_{i}=F_{i}\left(X_{i}\right)+\varepsilon G_{i}\left(X_{1}, \ldots, X_{n}, \varepsilon\right), \quad X_{i} \in \mathbb{R}^{m}, \quad i=1, \ldots, n, \quad \varepsilon \ll 1,
$$

such that each uncoupled subsystem

$$
\dot{X}_{i}=F_{i}\left(X_{i}\right)
$$

has an exponentially stable quasi-periodic attractor $M_{i}$. Then there is an $\varepsilon_{0}>0$ such that for all $\varepsilon \leq \varepsilon_{0}$ there is an open neighborhood $W$ of $M=M_{1} \times \cdots \times M_{n}$, and a continuous mapping $p: W \rightarrow \mathbb{T}^{\text {nk }}$ that transforms all local solutions of (2.1) to those of

$$
\dot{\theta}_{i}=\Omega_{i}+\varepsilon h_{i}\left(\theta_{1}, \ldots, \theta_{n}, \varepsilon\right), \quad \theta_{i} \in \mathbb{T}^{k}, \quad i=1, \ldots, n
$$

where each $\Omega_{i} \in \mathbb{R}^{k}$ is a nonresonant frequency vector, and $h_{i}$ is some function. Therefore, (2.3) is a local model for (2.1).

The proof of the theorem is given in Appendix B.

COROLlary 2.2. The phase model (2.3) is a canonical model for the whole family of brain models consisting of weakly connected quasi-periodic oscillators; see definitions in Appendix A.

We note that the phase model (2.3) has the same form as the canonical model for weakly connected limit cycle oscillators, with the only exception being that the phase variables $\theta_{i}$ and the frequencies $\Omega_{i}$ are vectors.

At this point the reader should wonder whether we gained any advantage converting (2.1) into (2.3). Indeed, to determine $h_{i}$ one needs knowledge about all of the 
functions $F_{i}$ and $G_{i}$ in (2.1), which is not available and probably will never be available. Thus, we cannot answer the question "What can system (2.3) do?" Nevertheless, using the theorem below we can answer the question, "What is it that system (2.3) cannot do regardless of the equations that describe the dynamics of each subsystem?" We show, in particular, that if the frequency vectors are chosen inappropriately, then no communication between elements of the system is possible regardless of the form of the connection functions $G_{i}$ and/or $h_{i}$.

3. Analysis of the phase model. Each phase vector $\theta_{i}(t)$ in the phase model (2.3) describes rhythmic activity of the $i$ th quasi-periodic oscillator. Obviously, the input from other oscillators is negligible on the time scale of order 1, since it has small magnitude $\varepsilon$. Nevertheless, such small inputs may accumulate and become significant on time scale of order $1 / \varepsilon$. Studying (2.3) on the time scale longer than $1 / \varepsilon$ involves the KAM (Kolmogorov-Arnold-Moser) theory and goes beyond the scope of this paper.

Analogous to the case of the phase model for weakly connected limit cycle oscillators (Theorems 9.7 and 9.9 in [19]), we prove the following result.

THEOREM 3.1. Consider the phase model (2.3), which we rewrite in the form

$$
\dot{\theta}=\Omega+\varepsilon h(\theta, \varepsilon), \quad \theta \in \mathbb{T}^{n k},
$$

where $\Omega=\left(\Omega_{1}, \ldots, \Omega_{n}\right) \in \mathbb{R}^{n k}$ is the combined vector of frequencies, and suppose that $h=\left(h_{1}, \ldots, h_{n}\right)$ has an absolutely convergent Fourier series. Then there is an $\varepsilon_{0}>0$ such that for all $\varepsilon \leq \varepsilon_{0}$ and all $t$ on a time scale of order $1 / \varepsilon$ there is a near identity change of variables

$$
\theta(t)=\vartheta(t)+o(1)
$$

that transforms (3.1) into a simpler model, which depends on the frequency vector $\Omega$.

- Nonresonant $\Omega$. If the combined frequency vector $\Omega$ is nonresonant (i.e., $l \cdot \Omega \neq 0$ for any nonzero $\left.l \in \mathbb{Z}^{n k}\right)$, then

$$
\dot{\vartheta}=\Omega+\varepsilon \omega+o(\varepsilon)
$$

for some constant $\omega \in \mathbb{R}^{n k}$.

- Resonant $\Omega$. Let $K$ be the resonant matrix of the frequency vector $\Omega$ (see the definition in section 1.5). Let $s$ be the number of rows of $K$. Then there is an analytic function $H$ defined on a lower-dimensional torus $\mathbb{T}^{s}=K \mathbb{T}^{n k}$ such that

$$
\dot{\vartheta}=\Omega+\varepsilon H(K \vartheta)+o(\varepsilon) .
$$

In any case, from (3.2) it follows that the activity of (3.1) is described accurately by either (3.3) or (3.4) on the large time scale of order $1 / \varepsilon$.

The proof is provided in Appendix C.

The following corollary is a straightforward consequence of Theorems 2.1 and 3.1.

Corollary 3.2. A solution $X(t)$ of the weakly connected system (2.1) is related to the solution $\vartheta(t)$ of the corresponding phase models (3.3) or (3.4) by the formula

$$
\vartheta(t)=p(X(t))+o(1)
$$

uniformly on a time interval of order $1 / \varepsilon$.

Thus, to understand locking behavior of (2.1) it suffices to study (3.3) and (3.4). 
3.1. Quasi-resonant relations. Strict resonance, $k \cdot \Omega=0$, is an abstract mathematical concept that may never be encountered in nature. It is more realistic to require that

$$
|k \cdot \Omega| \leq \epsilon
$$

for some small $\epsilon$. The condition above is referred to as being a quasi resonance. Obviously, all frequency vectors are quasi resonant for some $\epsilon \ll 1$, but only a few are of low order, that is, when $|k|$ is small.

Theorem 3.1 can easily be applied to quasi-resonant vectors. Let $\epsilon=\varepsilon$ and let $\Omega$ satisfy $|K \Omega| \leq \epsilon$ for some resonance matrix $K$. We represent $\Omega$ in the form

$$
\Omega=\Omega^{0}+\epsilon \Omega^{1} \quad \text { where } \quad K \Omega^{0}=0 \quad \text { and } \quad\left|K \Omega^{1}\right| \leq 1 .
$$

Now we rewrite system (3.1) in the form

$$
\dot{\theta}=\Omega^{0}+\varepsilon h^{0}(\theta, \varepsilon),
$$

where $h^{0}=h+\Omega^{1}$. Since $\Omega^{0}$ is resonant, the system above can be transformed into the form

$$
\dot{\vartheta}=\Omega^{0}+\varepsilon H^{0}(K \vartheta)+o(\varepsilon),
$$

which is an analogue of (3.4). Moreover, as it follows from the proof of Theorem 3.1, $H^{0}=H+\Omega^{1}$. Therefore, the system above can be written exactly in the form (3.4).

At this point the reader should wonder whether the resonances are important, since any nonresonant system can still be transformed into the form (3.4) using the definition of quasi resonance. However, (3.4) depends on the resonant matrix $K$. As was pointed out by Ermentrout ([9]; see also Proposition 9.14 in [19]), $H \rightarrow \omega$ when $|K| \rightarrow \infty$; that is, the function $H$ in (3.4) is almost a constant for high-order resonances. Therefore, only small-order (quasi) resonances would play a significant role in the dynamics of (3.3). How small the order should be depends upon $\varepsilon$.

COROllary 3.3. Low-order (high-order) quasi-resonant relations are qualitatively similar to the strict resonant (nonresonant) relations when $\epsilon$ has the same order as the strength of connections $\varepsilon$.

Without loss of generality we consider resonant and nonresonant frequency vectors below, but we tacitly imply that they can be low- or high-order quasi-resonant vectors, respectively. lems.

4. Applications. We apply Theorem 3.1 to mathematical neuroscience prob-

Let us consider the phase models (3.3) and (3.4) on time scales of order $1 / \varepsilon$, so that we can neglect the higher-order term $o(\varepsilon)$. First, we notice that one oscillator can "feel" the presence of another oscillator either through $\varepsilon \omega$ (in 3.3) or $\varepsilon H(K \vartheta)$ (in 3.4). The former describes the constant (averaged) influence that is due to the increased level of presynaptic excitation or inhibition. It does not depend on the state of the presynaptic oscillator. In contrast, the latter does. We say that oscillator $\vartheta_{j}$ affects or influences another oscillator $\vartheta_{i}$ if the behavior of the latter depends on the state $\vartheta_{j}(t)$. If in addition $\vartheta_{i}$ affects $\vartheta_{j}$, then we say that the oscillators communicate or interact.

We can carry these definitions over to the original system (2.1). We say that $X_{j}$ and $X_{i}$ interact if the state-dependent influences grow with time and become 
significant on the large time scale of order $1 / \varepsilon$. In contrast, if they remain small on such a large time scale, then the oscillators do not interact.

COROLLARY 4.1. If a weakly connected network of quasi-periodic oscillators in the form (2.1) or (2.3) does not have resonances, then the oscillators do not interact on the large time scale of order $1 / \varepsilon$. Any existing synaptic connections between the oscillators are functionally insignificant on such a time scale.

Proof. From Theorem 3.1 it follows that such a weakly connected system is governed by (3.3), which is uncoupled on the time scale of order $1 / \varepsilon$.

COROLlaRY 4.2. If a quasi-periodic oscillator, say, $\theta_{1}$, has a vector of frequencies, say, $\Omega_{1}$, that is not resonant with the combined vector of frequencies of the other oscillators, $\left(\Omega_{2}, \ldots, \Omega_{n}\right) \in \mathbb{R}^{(n-1) k}$, then $\theta_{1}$ cannot influence the other oscillators on the time scale of order $1 / \varepsilon$. It may, however, respond to other oscillators on this time scale.

That is, the oscillator can receive information, but it cannot transmit. Hence, the synaptic connections converging from other oscillators onto this one may be functionally significant, but synaptic connections diverging from this oscillator are functionally insignificant for any $h_{i}$.

Proof. Each row of the resonant matrix $K$ starts with $k$ zeros; that is, $K=\left(O, K_{1}\right)$ where $O$ is an $s \times k$ matrix of zeros, $K_{1}$ is some $s \times(n-1) k$ matrix, and $s$ is the number of rows. Therefore $K \theta$ in the system (3.4) does not depend on $\theta_{1}$.

Since each frequency vector $\Omega_{i}$ is nonresonant, any resonance relation in the combined vector $\left(\Omega_{2}, \ldots, \Omega_{n}\right) \in \mathbb{R}^{(n-1) k}$ would involve at least two oscillators. Therefore, $\theta_{1}$ cannot be affected by any of the oscillators $\theta_{2}, \ldots, \theta_{n}$ acting alone. There must be a combined effort of at least two such oscillators to affect the first one. A neurodynamical interpretation and significance of such a phenomenon have yet to be found. This phenomenon does not take place in the special case we discuss next.

4.1. Pairwise connections. Since we do not know the detailed mechanism of synaptic transmission, we have little information about the functions $G_{i}$ in (2.1). A reasonable assumption that arises frequently in applications is when each $G_{i}$ has a pairwise coupled form, at least up to order $\varepsilon$,

$$
G_{i}\left(X_{1}, \ldots, X_{n}, \varepsilon\right)=\sum_{j=1}^{n} G_{i j}\left(X_{i}, X_{j}\right)+\mathcal{O}(\varepsilon) .
$$

This assumption is a severe restriction and may not be satisfied in many cases. For example, if each variable $X_{i}$ describes activity of a single neuron, then the pairwise coupled form is not applicable to the following cases:

- The synaptic connections in the network are axo-axonic. In this case the transmission from $X_{j}$ to $X_{i}$ in Figure 4.1(a) may depend strongly on activity of another oscillator $X_{l}$, since firing of $X_{l}$ induces large amplitude inhibitory PSP in the presynaptic terminal of $X_{j}$. Such a PSP can alter significantly or even shut down the transmission through the synapse $X_{j} \rightarrow X_{i}$.

- Synaptic terminals are packed in tight glomeruli (as in Figure 4.1(b)) where different synapses may compete for scarce resources, such as extracellular calcium.

In contrast, if two synapses $X_{j} \rightarrow X_{i}$ and $X_{l} \rightarrow X_{i}$ are sufficiently far away from each other, as in Figure 4.1(c), then the pairwise coupled form (4.1) might be biologically plausible.

It is not clear what anatomical and physiological constraints may lead to the pairwise coupled form (4.1) when all the variables $X_{1}, \ldots, X_{n}$ describe dynamics of 


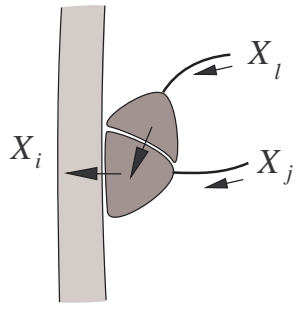

a

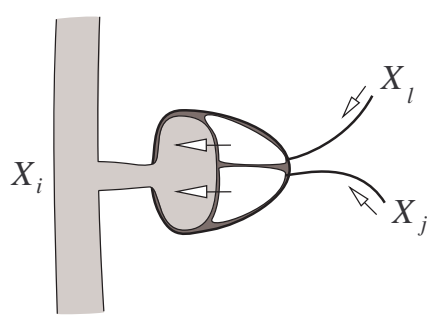

b

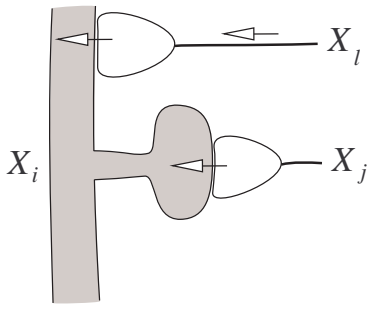

C

FIG. 4.1. (a) Transmission through the inhibitory synapse $X_{j} \rightarrow X_{i}$ can be shut down by the axo-axonic inhibitory synapse $X_{l} \rightarrow X_{j}$ (from [19]). (b) The synapses $X_{j} \rightarrow X_{i}$ and $X_{l} \rightarrow X_{j}$ are inside a synaptic glomerulus and may compete for limited extracellular resources. (c) Synaptic transmission $X_{j} \rightarrow X_{i}$ is relatively independent from $X_{l} \rightarrow X_{i}$, which may result in the pairwise coupled function (4.1).

cortical columns. It has been hypothesized [19] that if all synaptic connections between the columns are of the type depicted in Figure 4.1(c), then the pairwise coupled form (4.1) is biologically plausible. We stress that this is neither a fact nor a theorem, but a reasonable principle used in mathematical neuroscience, which still requires rigorous mathematical justification.

It is easy to check (see the discussion after Theorem 4.7 in [19]) that the pairwise coupled form of the functions $G_{i}$ leads to the pairwise coupled form of the functions $h_{i}$ in the phase model (2.3); that is,

$$
h_{i}\left(\theta_{1}, \ldots, \theta_{n}, \varepsilon\right)=\sum_{j=1}^{n} h_{i j}\left(\theta_{i}, \theta_{j}\right)+\mathcal{O}(\varepsilon) .
$$

This, in turn, leads to the pairwise coupled form of the functions $H_{i}$ in (3.4); that is,

$$
H_{i}(K \vartheta)=\sum_{j=1}^{n} H_{i j}\left(K_{i j}\left(\begin{array}{c}
\vartheta_{i} \\
\vartheta_{j}
\end{array}\right)\right)
$$

for some functions $H_{i j}$, where each $K_{i j}$ is the resonant matrix for the pair $\left(\Omega_{i}, \Omega_{j}\right) \in$ $\mathbb{R}^{2 k}$, and $H_{i j}=$ const when the pair is nonresonant. This implies the following refinement of Corollary 4.2.

Corollary 4.3. Consider pairwise weakly connected quasi-periodic oscillators of the form (2.1), (4.1). Any two such oscillators can communicate on the time scale of order $1 / \varepsilon$ only if they have mutually resonant frequency vectors.

In analogy with the case of weakly connected limit cycle oscillators, we say that interactions between weakly connected quasi-periodic oscillators are FM interactions. The interactions are most effective when the frequency vectors are equal or low-order resonant but not effective for high-order resonances.

4.2. The central element. Consider a weakly connected system of the form

$$
\begin{aligned}
\dot{X}_{0} & =F_{0}\left(X_{0}\right)+\varepsilon G_{0}\left(X_{0}, X_{1}, \ldots, X_{n}, \varepsilon\right), \\
\dot{X}_{i} & =F_{i}\left(X_{i}\right)+\varepsilon \sum_{j=1}^{n} G_{i j}\left(X_{0}, X_{i}, X_{j}, \varepsilon\right), \quad i=1, \ldots, n,
\end{aligned}
$$




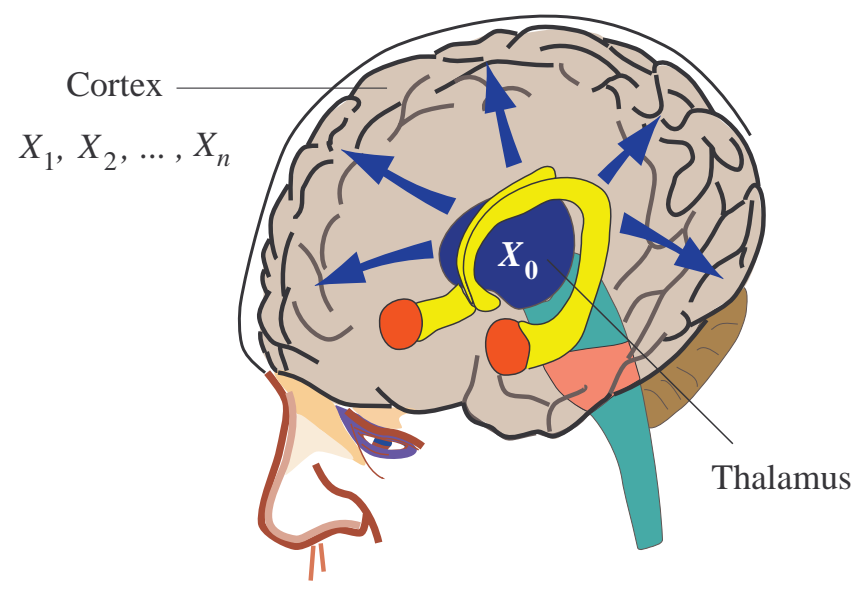

FIG. 4.2. System (4.5) may describe cortical oscillators forced by the thalamic input.

which describes the activity of a network of $n$ quasi-periodic oscillators $X_{1}, \ldots, X_{n}$ with a "central" element $X_{0}$. Such a system may describe cortical oscillators forced by the thalamic input $X_{0}$ (see Figure 4.2), or hippocampal oscillators forced by the septal input [21, 19]. Notice that synaptic connections within the network (4.5) are axo-dendritic, but connections from the central element to the network may be axoaxonic or form synaptic glomeruli so that $X_{0}$ may modulate the synaptic transmission in the network (see Figure 4.3).

The corresponding phase model has the form

$$
\begin{aligned}
\dot{\theta}_{0} & =\Omega_{0}+\varepsilon h_{0}\left(\theta_{0}, \theta_{1}, \ldots, \theta_{n}, \varepsilon\right), \\
\dot{\theta}_{i} & =\Omega_{i}+\varepsilon \sum_{j=1}^{n} h_{i j}\left(\theta_{0}, \theta_{i}, \theta_{j}, \varepsilon\right), \quad i=1, \ldots, n .
\end{aligned}
$$

Whether or not the $j$ th oscillator can interact with the $i$ th oscillator depends on the existence of resonances in the triple $\left(\Omega_{0}, \Omega_{i}, \Omega_{j}\right) \in \mathbb{R}^{3 k}$. In analogy with periodic oscillators (section 9.5.1 in [19]) we identify several interesting cases:

- If the combined vector $\left(\Omega_{0}, \Omega_{i}, \Omega_{j}\right)$ is nonresonant, then the $i$ th and the $j$ th oscillators do not interact on the time scale of order $1 / \varepsilon$.

- Suppose the combined vector $\left(\Omega_{0}, \Omega_{i}, \Omega_{j}\right)$ has a resonance relation involving only two frequency vectors. If $\Omega_{j}$ does not participate in such a relation, then the oscillators do not interact.

- If the combined vector $\left(\Omega_{0}, \Omega_{i}, \Omega_{j}\right)$ has a resonant relation involving all three frequency vectors, then the oscillators can interact.

The latter case is especially interesting since it may happen that the combined vector has a resonance relation even though $\Omega_{i}$ and $\Omega_{j}$ are mutually nonresonant. The simplest case when this happens corresponds to $\Omega_{0}=\left|\Omega_{i} \pm \Omega_{j}\right|$, which we illustrate in Figure 5.6.

Corollary 4.4. A weak input from the central element may dynamically link two quasi-periodic oscillators having mutually nonresonant frequency vectors $\Omega_{i}$ and $\Omega_{j}$ provided that the input frequency $\Omega_{0}$ is resonant with the combined frequency $\left(\Omega_{i}, \Omega_{j}\right)$.

Thus, changing its pattern of rhythmic activity (i.e., the frequency vector $\Omega_{0}$ ), the 


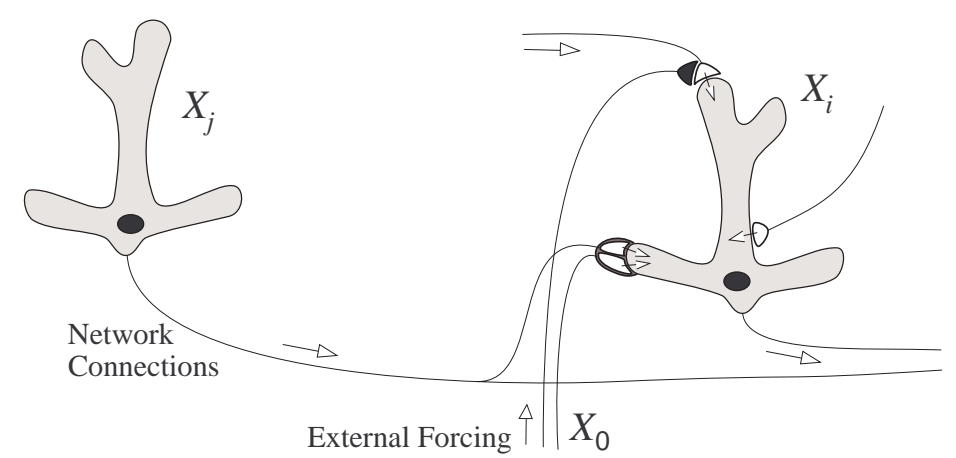

FIG. 4.3. System (4.5) describes a network of pairwise connected oscillators. External forcing $X_{0}$ may modulate the synaptic transmission in the network via axo-axonic synapses or synaptic glomeruli or both.

thalamus may have complete control over the information processing taking place in the cortex. It may dynamically link any two cortical columns, even those that oscillate with mutually nonresonant frequencies and would have otherwise been unlinked.

4.3. Identical frequencies. We continue to study the phase model (2.3) with the connection functions of the form (4.2). Every pair $\left(\Omega_{i}, \Omega_{j}\right)$ may have up to $k$ resonances. Each resonant relation involves entries from both vectors. Indeed, the converse would imply that either $\Omega_{i}$ or $\Omega_{j}$ is a resonant vector, which would contradict Theorem 2.1. An important case when each pair has exactly $k$ simple resonant relations is considered below.

COROLLARY 4.5. If pairwise weakly coupled quasi-periodic oscillators have identical frequency vectors $\Omega_{i}$, then the phase model can be transformed into the form

$$
\dot{\vartheta}_{i}=\Omega_{i}+\varepsilon \sum_{j=1}^{n} H_{i j}\left(\vartheta_{j}-\vartheta_{i}\right) .
$$

Moreover, if we use the slow time $\tau=\varepsilon t$ and the phase deviation variables $\varphi(\tau)=$ $\vartheta(t)-\Omega t$, then the system above can be written in the form

$$
\varphi_{i}^{\prime}=\sum_{j=1}^{n} H_{i j}\left(\varphi_{j}-\varphi_{i}\right),
$$

where' $=d / d \tau$.

Proof. Each pair $\left(\Omega_{i}, \Omega_{j}\right) \in \mathbb{R}^{2 k}$ has precisely $k$ resonance relations $\Omega_{j l}-\Omega_{i l}=0$ for $l=1, \ldots, k$. The corresponding resonant matrix has the form

$$
K=\left(\begin{array}{cccccc}
-1 & \cdots & 0 & 1 & \cdots & 0 \\
\vdots & \ddots & \vdots & \vdots & \ddots & \vdots \\
0 & \cdots & -1 & 0 & \cdots & 1
\end{array}\right)
$$

that is, $K=(-I, I)$, where $I$ is the identity $k \times k$ matrix. Therefore,

$$
K\left(\begin{array}{c}
\vartheta_{i} \\
\vartheta_{j}
\end{array}\right)=\vartheta_{j}-\vartheta_{i}
$$


for any $i$ and $j$. Then the proof follows from (4.3).

System (4.6) suggests that interactions between weakly connected quasi-periodic oscillators occur via phase deviations. Electrical engineers refer to such interactions as being phase modulation (PM) interactions. From a mathematical point of view $\mathrm{PM}$ is the same as FM, which suggests the term FM interactions.

4.4. Kuramoto's model. It is customary in the physics literature to keep the initial portion of the Fourier series of the functions $H_{i j}$ and disregard the rest. In the case of weakly connected limit cycle oscillators this approximation leads to Kuramoto's model [24], which is canonical for weakly connected class 1 excitable oscillators having delayed interactions [20] and for weakly connected Andronov-Hopf oscillators. Applying the same procedure to system (4.6) yields

$$
\varphi_{i}^{\prime}=\omega_{i}+\sum_{j=1}^{n} s_{i j} \sin \left(\varphi_{j}+\psi_{i j}-\varphi_{i}\right), \quad i=1, \ldots, n,
$$

where $\omega_{i} \in \mathbb{R}^{k}$ is the vector of center frequencies of the $i$ th quasi-periodic oscillator, and each term

$$
s_{i j} \sin \left(\varphi_{j}+\psi_{i j}-\varphi_{i}\right)=\left(\begin{array}{c}
s_{i j 1} \sin \left(\varphi_{j 1}+\psi_{i j 1}-\varphi_{i 1}\right) \\
s_{i j 2} \sin \left(\varphi_{j 2}+\psi_{i j 2}-\varphi_{i 2}\right) \\
\vdots \\
s_{i j k} \sin \left(\varphi_{j k}+\psi_{i j k}-\varphi_{i k}\right)
\end{array}\right)
$$

is a column vector. The fundamental difference between the phase model (4.6) and the generalization of the Kuramoto model above is that there may be interactions between different oscillatory modes in (4.6), but there is no such interference in the generalized Kuramoto model. Indeed, the latter is partitioned into $k$ disjoint subnetworks of Kuramoto type

$$
\varphi_{i l}^{\prime}=\omega_{i l}+\sum_{j=1}^{n} s_{i j l} \sin \left(\varphi_{j l}+\psi_{i j l}-\varphi_{i l}\right), \quad i=1, \ldots, n,
$$

for $l=1, \ldots, k$, which can be studied separately. Obviously, (4.6) does not allow such a partition unless we impose additional restrictions onto the functions $H_{i j}$.

The generalized Kuramoto model provides a clear example of multiplexing of signals: A pair of oscillators can communicate via $k$ independent channels using a single transmission line. The channels are the components of the vectors $\varphi_{i} \in \mathbb{T}^{k}$, and the number of transmission lines is the dimension of the set $G_{i j}\left(M_{i}, M_{j}\right)$. There is only one transmission line when $G_{i j}\left(X_{i}, X_{j}\right)=\vec{v} \tilde{G}_{i j}\left(X_{i}, X_{j}\right)$ for some nonzero vector $\vec{v} \in \mathbb{R}^{m}$ and a scalar function $\tilde{G}_{i j}: \mathbb{R}^{2 m} \rightarrow \mathbb{R}$. We consider an example of this in section 5.3.

5. Examples. To illustrate the theory developed above we consider a pair of weakly connected cortical columns having quasi-periodic autonomous activity. We model each column by a collection of periodic oscillators, each having different frequencies. For this purpose we use well-known Wilson-Cowan oscillators (in section 5.2 ) and leaky integrate-and-fire oscillators (in section 5.3). The former gives a smooth system satisfying the conditions of Theorems 2.1 and 3.1, while the latter gives a discontinuous (pulse-coupled) system [20]. It is remarkable that our theory still provides an accurate description of behavior of such a pulse-coupled system. 


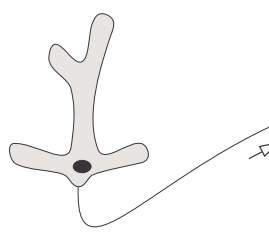

Neuron 1

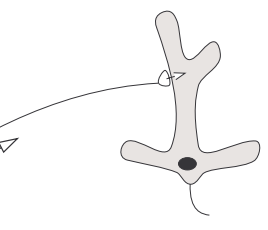

Neuron 2
Neuron 1 | ||||| | | || |||||| | | |||||||| | |||||||

Neuron $2-||||||||||||||||||||||||||||||||||||$

What does neuron 1 tell neuron 2 ?

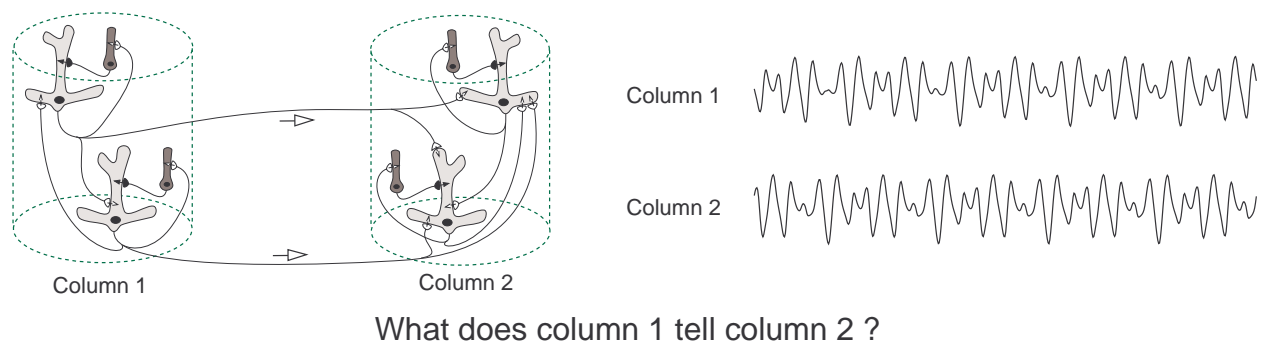

Fig. 5.1. The nature of neural code: "What does neuron (column) 1 tell neuron (column) 2?" We study a simpler question: "Do neurons (columns) 1 and 2 talk?" See details in the text.

Our choice of the models is purely subjective. We count on their simplicity and popularity but not on their biological plausibility.

5.1. Neural code. One of the most intriguing problems in neuroscience is determining the nature of neural code: What does neuron (column) 1 tell neuron (column) 2 in Figure 5.1? How are the neural signals encoded? What should neuron (column) 2 do with the incoming signal to make sense of it? Is the information hidden in the mean firing rate, in the interspike interval, or in something else?

In this paper we study a simpler question: "Do neurons (columns) 1 and 2 in Figure 5.1 communicate?" The theory developed above provides a partial answer: If the neurons (columns) are weakly connected and they exhibit quasi-periodic (multifrequency) rhythmic activity with frequency vectors $\Omega_{1}$ and $\Omega_{2}$, then communication occurs only when the vectors have low-order mutual resonances. Since we use the canonical model approach, the result does not depend on whether we simulate Wilson-Cowan, Hodgkin-Huxley, Miles-Traub, or any other neural model.

Phase resetting experiment. Looking at the spike trains in Figure 5.1, it is difficult to determine whether or not the neurons interact. Indeed, it is not clear whether each spike of the postsynaptic neuron is due to its intrinsic properties or to input to it. The same difficulty arises in the case of cortical columns, since it is not apparent what features of the curves are due to autonomous oscillatory properties of the cortical columns and what are due to weak interactions. To reveal the interactions, we employ the following phase resetting experiment: We perform two simulations with the same parameters and initial conditions, but during the second simulation we apply a brief strong stimulus to the first column to advance its phase. The new (perturbed) solutions are plotted as dashed curves in Figure 5.2, or dashed bars in Figures 5.4-5.7. If the second column "feels" the perturbation, then its dashed solution would differ significantly from the continuous one. This simulation experiment is carried out here 

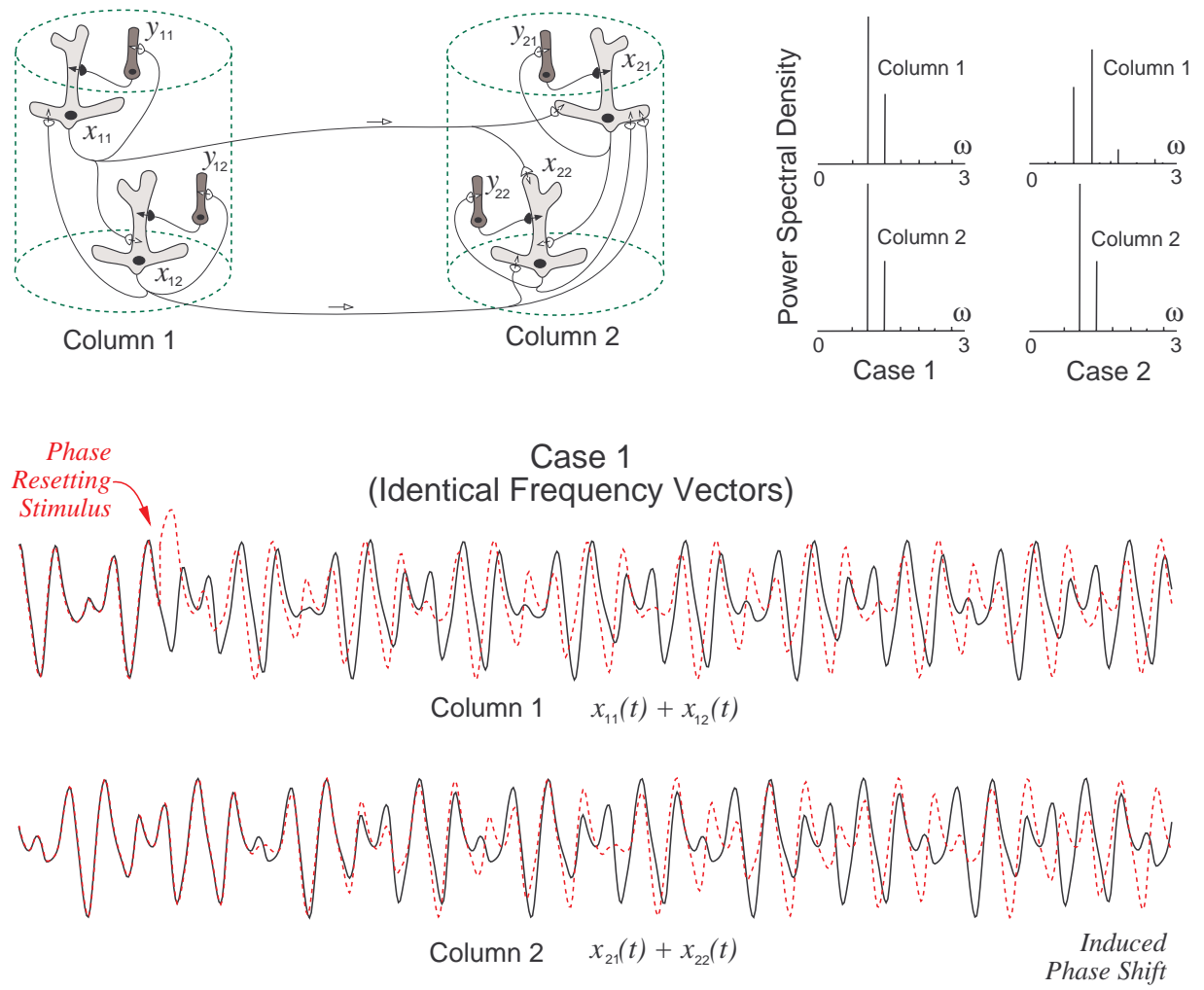

Case 2

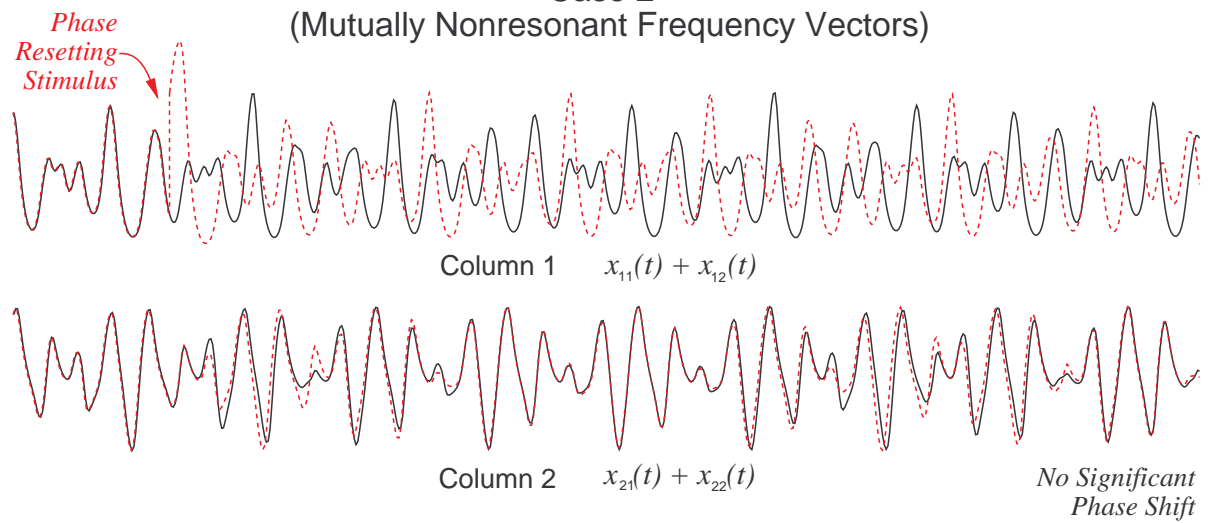

FIG. 5.2. Frequency modulated (FM) interactions: Interactions between two cortical columns (top) exhibiting quasi-periodic activity depend on their frequency vectors. Case 1: Identical frequency vectors. If a brief strong stimulus is applied to Column 1 to change its phase (dashed curve), Column 2 acquires a phase shift too. Therefore, the columns interact through phases. Case 2: Nonresonant frequency vectors. Column 2 is relatively insensitive to any changes of phase of Column 1. Therefore, the columns do not interact, even though they are connected. Parameters: Each column consists of two strongly connected Wilson-Cowan neural oscillators of the form (5.1) with $a=b=c=10, d=-2, \rho_{\mathrm{y}}=-6, \rho_{\mathrm{x} 11}=\rho_{\mathrm{x} 21}=-2, \rho_{\mathrm{x} 22}=2$, and $\rho_{\mathrm{x} 12}=2$ (in Case 1) or $\rho_{\mathrm{x} 12}=-3$ (in Case 2), $\varepsilon=1 / 20, t \in[0,170]$. Stimulus: The state of Column 1 is reset to $\left(x_{11}, y_{11}, x_{12}, y_{12}\right)=(0.7,-0.7,0.7,-0.7)$ at $t=35$. 
for both the Wilson-Cowan and integrate-and-fire models.

5.2. Wilson-Cowan oscillators. We consider two weakly connected cortical columns modeled by a pair of strongly connected neural oscillators of the WilsonCowan type $[45,46,19]$. Let $i=1,2$ be the column number and $j=1,2$ be the oscillator number within the column. Each neural oscillator is described by the vector $\left(x_{i j}, y_{i j}\right) \in \mathbb{R}^{2}$, where $x_{i j}$ and $y_{i j}$ denote activities of local population of excitatory and inhibitory neurons, respectively. Each such population is depicted as a single "averaged" neuron in Figure 5.2.

There could be many synaptic organizations within each column and between the columns $[6,18]$. For our simulations we choose the simplest one: The neural oscillators are connected through excitatory neurons. Thus, we consider the model

$$
\left\{\begin{array}{l}
\dot{x}_{i j}=-x_{i j}+S\left(\rho_{\mathrm{x} i j}+a x_{i j}-b y_{i j}+E_{i j}\right), \\
\dot{y}_{i j}=-y_{i j}+S\left(\rho_{\mathrm{y}}+c x_{i j}-d y_{i j}\right)
\end{array}\right.
$$

where $i=1,2, \quad j=1,2$;

$$
S(\rho)=\frac{1}{1+e^{-\rho}}
$$

is an S-shaped function; and $E_{i j}$ is the input from other oscillators

$$
\begin{array}{cc}
\text { Column } 1 & \text { Column } 2 \\
E_{11}=x_{12}+\varepsilon\left(x_{21}+x_{22}\right), & E_{21}=x_{22}+\varepsilon\left(x_{11}+x_{12}\right), \\
E_{12}=x_{11}+\varepsilon\left(x_{21}+x_{22}\right), & E_{22}=x_{21}+\varepsilon\left(x_{11}+x_{12}\right) .
\end{array}
$$

In all our simulations we use $a=b=c=10, d=-2, \rho_{\mathrm{y}}=-6$, and $\varepsilon=1 / 20$. If we take identical $\rho_{\mathrm{x} i j}$, then the neural oscillators within each column synchronize, and the column activity is periodic. Other choices for $\rho_{\mathrm{x} i j}$ lead to either $p: q$ frequency locking $[9,19]$, quasi-periodic, or chaotic activity.

5.2.1. Identical frequency vectors. First, we consider the case of identical columns. The choice $\rho_{\mathrm{x} i 1}=-2$ and $\rho_{\mathrm{x} i 2}=2, i=1,2$, results in quasi-periodic column activity. Its power spectrum is discrete (see top of Figure 5.2) with the highest peaks corresponding to the entries of the frequency vectors $\Omega_{1}=\Omega_{2} \approx(1.0426,1.3615) \in \mathbb{R}^{2}$.

We plot the activity of each column, $x_{i 1}(t)+x_{i 2}(t)$, as a continuous curve in the middle of Figure 5.2. Looking at the curves it is hard to determine whether or not the columns interact. Indeed, it is not clear what features of the curves are due to intrinsic dynamical properties of the columns and what are due to the weak interactions. To reveal the interaction, we change the phase of the first column by applying a brief strong stimulus and plot the new (perturbed) solutions as dashed curves. Now one can see that the second column acquires a phase shift. Since it starts from the same initial condition, the induced phase shift is due to the weak interactions, which agrees with Corollary 4.3.

5.2.2. Mutually nonresonant frequency vectors. Now consider the case when the cortical columns have quasi-periodic dynamics with different frequency vectors. For this we set $\rho_{\mathrm{x} 12}=-3$ and leave the other parameters unchanged. The frequency vector of the second column, $\Omega_{2} \approx(1.0426,1.3615) \in \mathbb{R}^{2}$, is unchanged, but the frequency vector of the first column becomes $\Omega_{1} \approx(0.9077,1.2756) \in \mathbb{R}^{2}$. The combined frequency vector $\Omega=\left(\Omega_{1}, \Omega_{2}\right) \in \mathbb{R}^{4}$ does not have low-order resonances. Surprisingly, a quasi-resonant relation $(3,0,0,-2) \cdot \Omega \approx 0.0001$ having order 5 did not play a significant role in our simulations. 


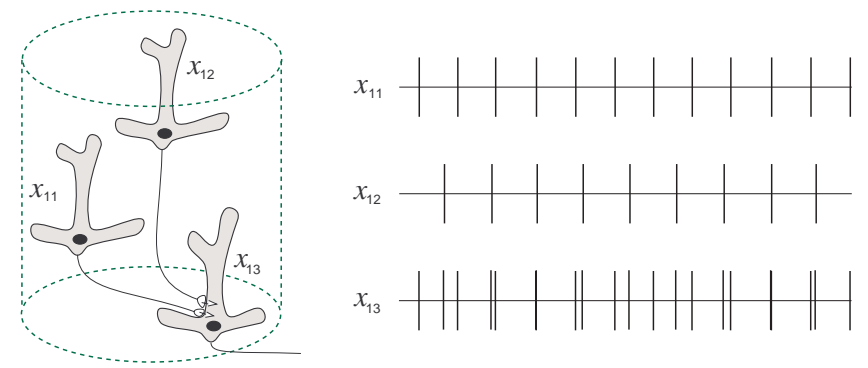

FIG. 5.3. Integrate-and-fire projection neuron $x_{13}$ receives very strong input from integrate-andfire local-circuit oscillators $x_{11}$ and $x_{12}$ having incommensurable frequencies $\omega_{11}$ and $\omega_{12}$. Therefore, the column output $x_{13}(t)$ exhibits quasi-periodic spiking activity with the frequency vector $\Omega_{1}=$ $\left(\omega_{11}, \omega_{12}\right)$.

The result of the phase resetting experiment described above is depicted at the bottom of Figure 5.2. We see that the induced phase shift of the second cortical column remains negligible even on a long time scale. This implies that even though the columns are interconnected, the second column is not sensitive to phase of the first one, which corroborates Corollary 4.1.

5.3. Integrate-and-fire neurons. Let us model each cortical column $(i=1,2)$ by three leaky integrate-and-fire neurons (see Figure 5.3). Each such neuron has the form $[34,31,23,20]$

$$
\dot{x}=a-x+\varepsilon E(t),
$$

where $x \in[0,1]$ is its "voltage," $E(t)$ is the input from the other neurons, and $a$ is a parameter. When $x$ crosses $x=1$, the oscillator is said to fire a spike and $x$ is reset to $x=0$. At this moment the voltages of the other integrate-and-fire neurons are incremented by $\varepsilon$. To stress this fact we say that the neurons are pulse coupled and we write

$$
E(t)=\sum_{l=-\infty}^{l=+\infty} \delta\left(t-t^{l}\right)
$$

where each $t^{l}$ is the time of presynaptic firing and $\delta$ is the Dirac delta function.

If $a>1$ and $E(t) \equiv 0$, then the leaky integrate-and-fire neuron exhibits periodic activity with the frequency

$$
\omega=-\frac{2 \pi}{\ln (1-1 / a)} .
$$

(Some mammalian cortical neurons can exhibit intrinsic oscillatory activity in vitro in the $10-$ to $50-\mathrm{Hz}$ frequency range $[25,26]$.)

Quasi-periodic activity can result from two such integrate-and-fire oscillators having incommensurable frequencies $\omega_{i 1}$ and $\omega_{i 2}$ and making very strong synaptic connection to another integrate-and-fire neuron (see Figure 5.3) so that $x_{i 3}$ fires whenever $x_{i 1}$ or $x_{i 2}$ does. Since the spike train of $x_{i 3}$ is a superposition of the spike trains of $x_{i 1}$ and $x_{i 2}$, the projection neuron $x_{i 3}(t)$ (and hence the entire cortical column) has quasiperiodic spiking activity with a nonresonant frequency vector $\Omega_{i}=\left(\omega_{i 1}, \omega_{i 2}\right) \in \mathbb{R}^{2}$. 

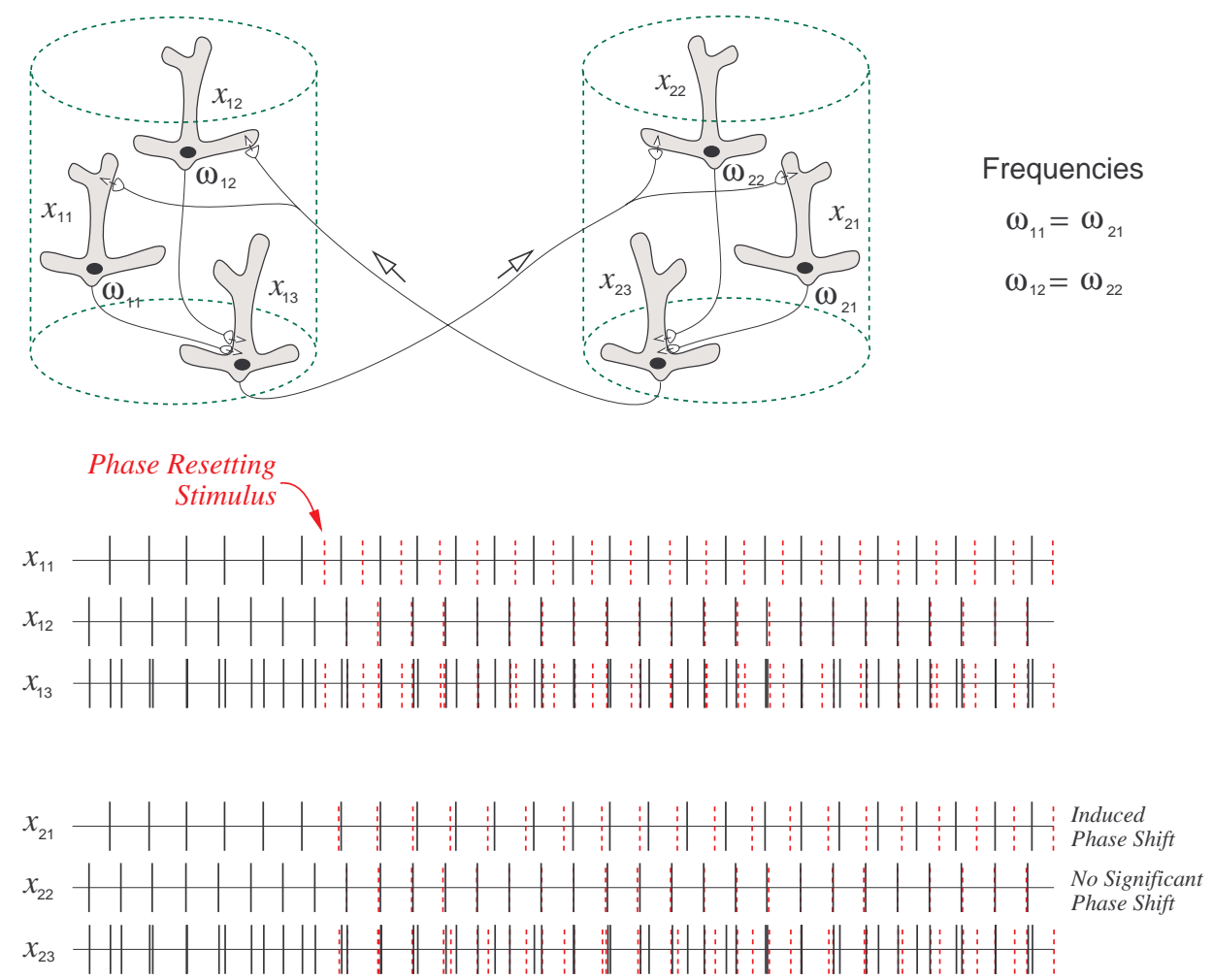

FIG. 5.4. Multiplexing of signals. Interaction between identical neurons $x_{11}$ and $x_{21}$ does not interfere with the interaction between the neurons $x_{12}$ and $x_{22}$ having different frequencies even though all neurons use a single transmission line $x_{13}$. When we change the phase of $x_{11}$ by applying a phase resetting stimulus, the corresponding neuron $x_{21}$ acquires a phase shift, but $x_{22}$ does not (compare continuous and dashed bars). Parameters: Each column consists of strongly connected leaky integrate-and-fire neurons (5.2) with $a_{11}=a_{21}=2, a_{21}=a_{22}=2.3$, and $a_{13}=a_{23}=0$; $\varepsilon=1 / 20$ and $t \in[0,20]$. Stimulus: Variable $x_{11}$ is reset to $x_{11}=1$ at $t=4$.

Before proceeding any further, we warn the reader that such a spiking quasiperiodic cortical column is just a caricature that might have nothing in common with biological reality. The major reason we use it is to show that our theory can be applied to discontinuous (pulse-coupled) systems (see also [20]). Besides, the column architecture provides an easy way to control the frequency vector.

5.3.1. Identical columns: Multiplexing of signals. Let us consider identical weakly pulse-coupled cortical columns described by leaky integrate-and-fire neurons (5.2) with $a_{11}=a_{21}=2, a_{21}=a_{22}=2.3$, and $a_{13}=a_{23}=0$. We take $\varepsilon=1 / 20$ to be the strength of connections between the columns.

Both neurons $x_{21}$ and $x_{22}$ in the second column receive an identical quasi-periodic spike train from the first column via $x_{13}(t)$; see the top of Figure 5.4. Can $x_{21}$ distinguish between the part of the signal coming from $x_{11}$ and the part coming from $x_{12}$ ? To find out we perturb the phase of the first neuron without changing the phase of the second one. The old (new) activity is depicted as continuous (dashed) bars in Figure 5.4. 


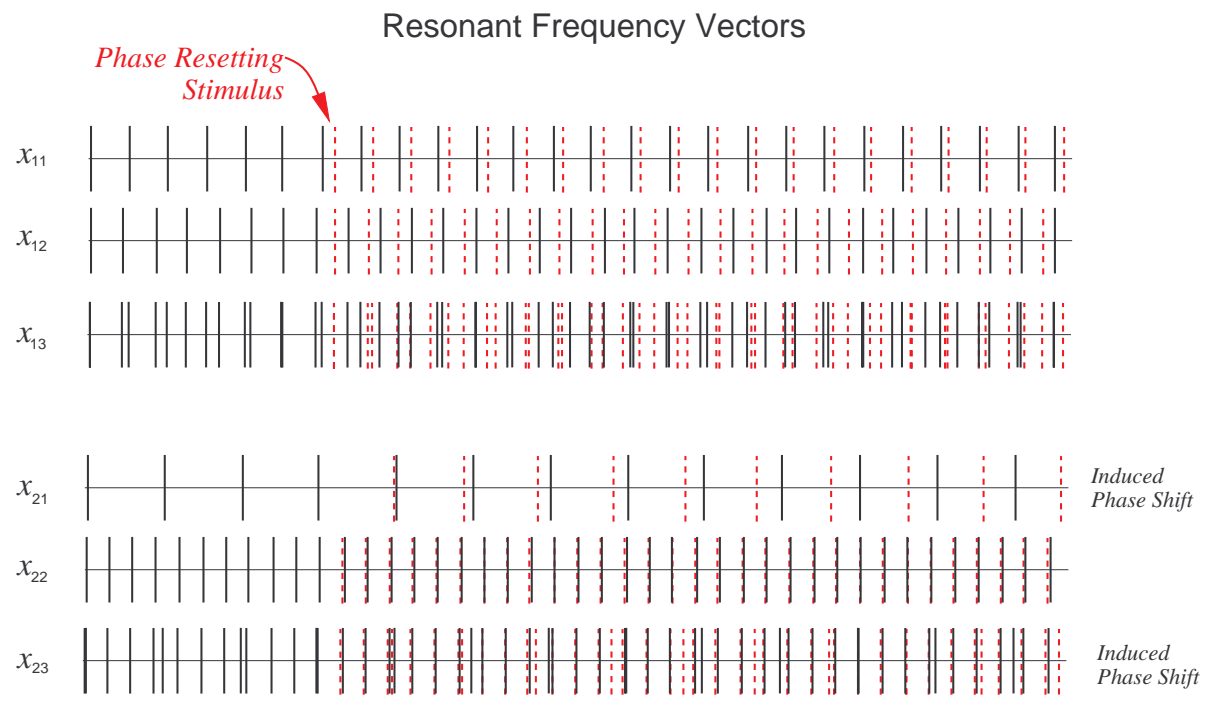

Non-Resonant Frequency Vectors
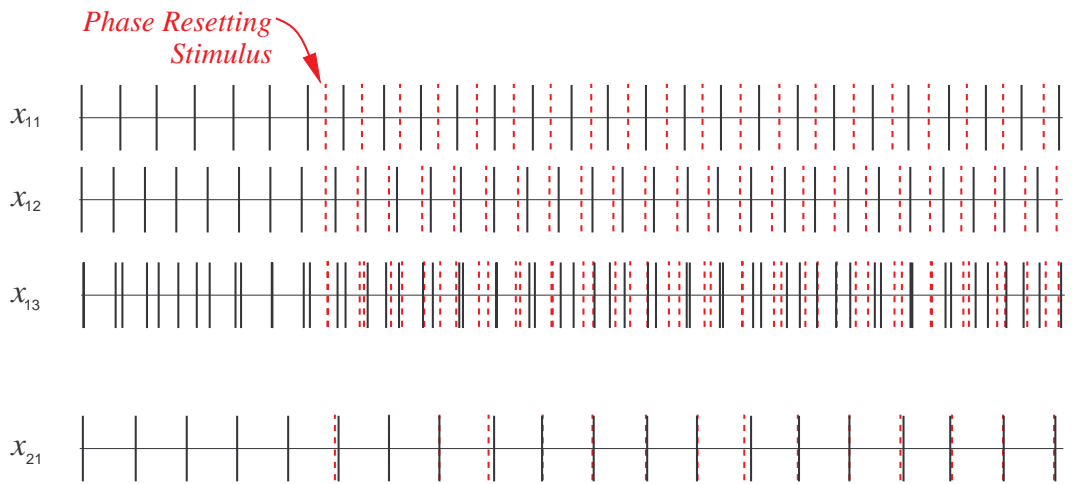

$x_{22}$

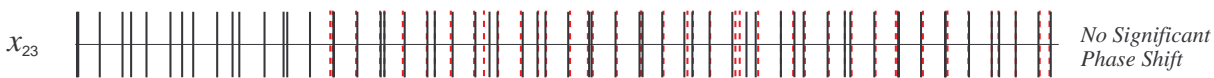

FIG. 5.5. Frequency modulated (FM) interactions. Interactions between two cortical columns from Figure 5.4 exhibiting quasi-periodic spiking activity depend on their frequency vectors $\Omega_{1}=$ $\left(\omega_{11}, \omega_{12}\right)$ and $\Omega_{2}=\left(\omega_{21}, \omega_{22}\right)$. Top: Frequency vectors have a resonance $(2,-2,1,0) \cdot\left(\Omega_{1}, \Omega_{2}\right)=0$; that is, $\omega_{21}=2\left(\omega_{12}-\omega_{11}\right)$. Notice that the second column can distinguish the phase of the first one. Bottom: Nonresonant frequency vectors. The second column cannot distinguish the phase of the first one. Parameters: $\varepsilon=1 / 20, a_{11}=2, a_{12}=2.3, a_{22}=3$. The choice $a_{21} \approx 1.25$ produces resonant frequency vectors and $a_{21}=1.6$ produces nonresonant (or high-order resonant) ones. 

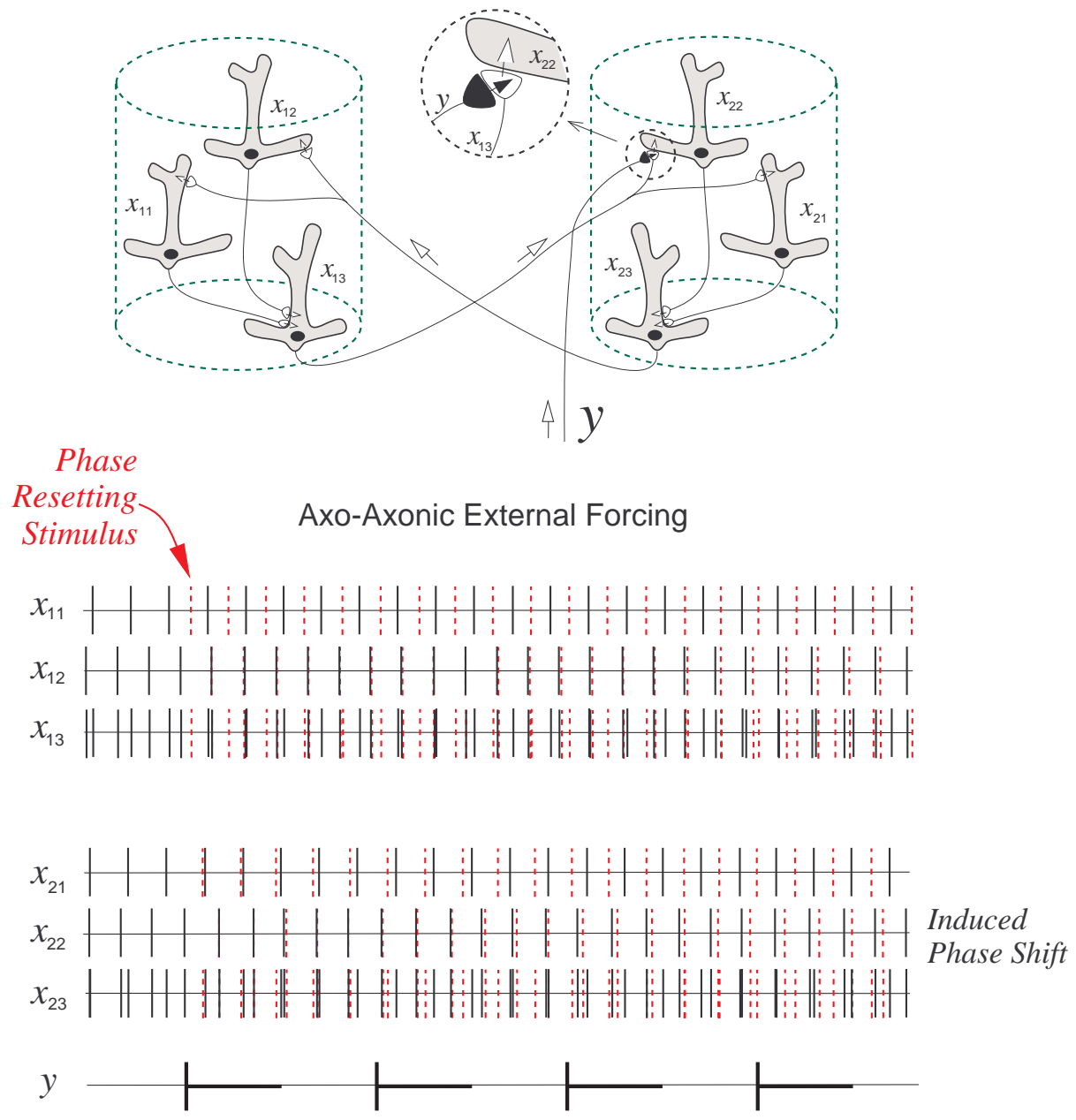

FIG. 5.6. Axo-axonic periodic input $y(t)$ with the frequency $\omega_{\mathrm{y}}=\omega_{22}-\omega_{11}$ can revive communication between $x_{11}$ and $x_{22}$ having mutually nonresonant frequencies. All parameters are as in Figure 5.4.

From the figure one can see that $x_{21}$ acquires a phase shift while $x_{22}$ does not. If we changed the phase of $x_{12}$, then $x_{21}$ would not acquire a phase shift but $x_{22}$ would. Thus, the pairs of neurons $x_{11}, x_{21}$ and $x_{12}, x_{22}$ oscillating with different frequencies can communicate selectively using different channels but using a single transmission line. This is probably the simplest example of multiplexing of neural signals.

5.3.2. Resonant frequency vectors. Now we consider the case when cortical columns have mutually resonant frequency vectors $\Omega_{1}=\left(\omega_{11}, \omega_{12}\right)$ and $\Omega_{2}=$ $\left(\omega_{21}, \omega_{22}\right)$. At the top of Figure 5.5 we simulate two such columns having resonant relation $(2,-2,1,0) \cdot\left(\Omega_{1}, \Omega_{2}\right)=0$; that is, $\omega_{21}=2\left(\omega_{12}-\omega_{11}\right)$. The values of parameters $a_{i j}$ that result in such a resonance relation can easily be found using (5.3).

Since all neurons have pairwise incommensurable frequencies, one would expect that each neuron from the second column would be insensitive to any phase changes of any neuron from the first column. However, the phase resetting test shows that this is 


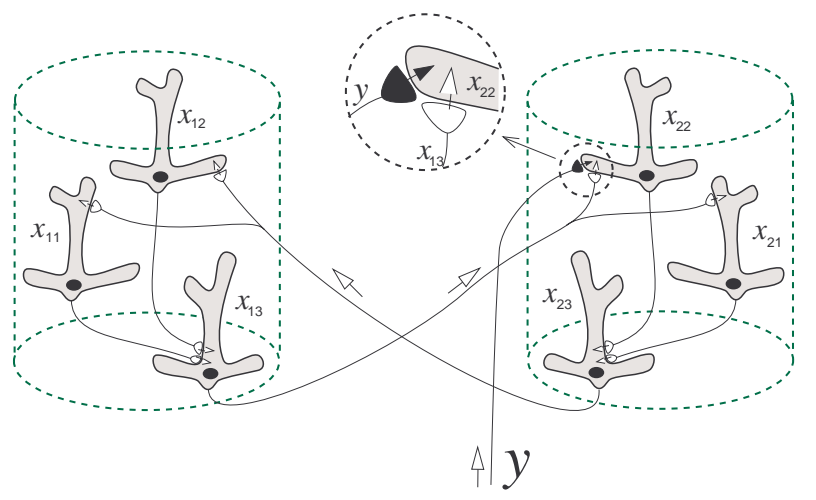

Phase

Resetting Stimulus

Axo-Dendritic External Forcing

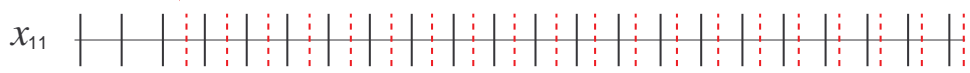

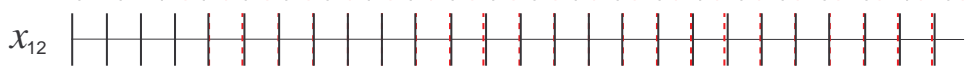

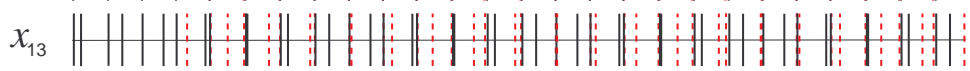

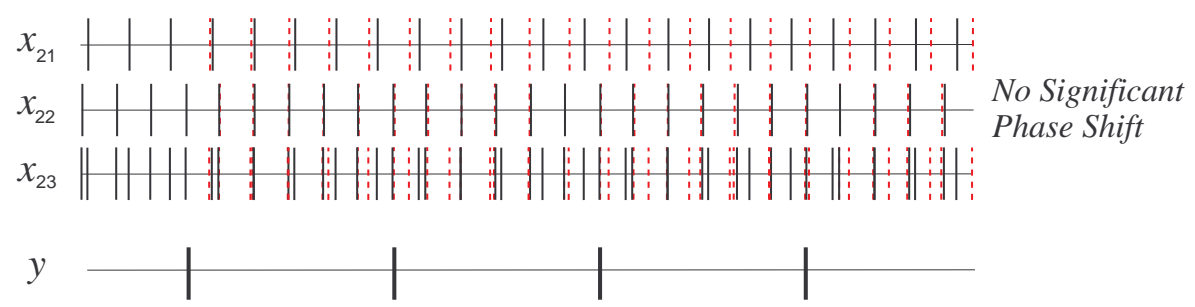

FIG. 5.7. Axo-dendritic input cannot dynamically link noninteracting oscillators $x_{11}$ and $x_{22}$. All parameters are as in Figure 5.4.

not the case: If we change the phase of the first column, the second column (variable $x_{23}$ ) acquires a phase shift. This happens because the quasi-periodic spike train of $x_{13}$ has not only the frequencies $\omega_{11}$ and $\omega_{12}$, but also their linear combinations including $2\left(\omega_{12}-\omega_{11}\right)$ which equals $\omega_{21}$. Thus, $x_{21}$ can extract whatever is transmitted on this frequency combination.

If we change the parameters so that the frequency vectors become nonresonant, then no interaction between the cortical columns is possible (see the lower part of Figure 5.5). In any case, the behavior of weakly pulse-coupled cortical columns is described accurately by Corollary 4.3.

5.3.3. Axo-axonic input. Let us return to the case of identical oscillators in which $x_{11}$ communicates with $x_{21}$ and $x_{12}$ communicates with $x_{22}$ without any cross interference (see Figure 5.4). Suppose there is an external periodic input $y$ that modulates the synaptic transmission between "noncommunicating" neurons $x_{11}$ and $x_{22}$ via axo-axonic synapses as we depict in Figure 5.6. We assume that $y$ fires with 
frequency $\omega_{\mathrm{y}}$ and the synaptic transmission from $x_{13}$ to $x_{22}$ is blocked during half a period after each firing of $y$; see bold bars at the bottom of Figure 5.6. If the triple $\left(\omega_{\mathrm{y}}, \omega_{11}, \omega_{22}\right)$ is resonant, then Corollary 4.4 suggests that $x_{11}$ could influence $x_{22}$.

In our simulations depicted in Figure 5.6 we chose $\omega_{\mathrm{y}}=\omega_{22}-\omega_{11}$ and perform the phase resetting experiment. Our simulations corroborate Corollary 4.4 in that a periodic axo-axonic input $y(t)$ dynamically links $x_{11}$ and $x_{22}$ even though they oscillate with mutually nonresonant frequencies and would have been unlinked otherwise.

Finally, we perform exactly the same experiment, but with axo-dendritic inhibitory input $y(t)$; see Figure 5.7. Every time $y$ fires, the membrane potential of $x_{22}$ is decreased by $\varepsilon$. The effect depicted in Figure 5.6 disappears, which means that the external input must modulate the synaptic transmission in the network in order to dynamically link any noninteracting oscillators; see section 4.2 for more details.

6. Discussion. In this paper we generalize the theory of weakly connected periodic (limit cycle) oscillators to include the case of quasi-periodic oscillations. In either case the dynamics of the network can be transformed into the phase model (2.3) by a continuous noninvertible change of variables. The only difference between periodic and quasi-periodic oscillators is that each $\theta_{i}$ belongs to the unit circle $\mathbb{S}^{1}$ in the former case, and to the $k$-torus $\mathbb{T}^{k}$ in the latter case.

Our approach is not to determine what oscillatory networks can accomplish, but to determine what oscillatory networks cannot accomplish regardless of the equations that describe their dynamics.

6.1. FM interactions. We find that whether or not weakly connected oscillators can communicate depends on the relation between their frequency vectors $\Omega_{i} \in \mathbb{R}^{k}$. When the vectors are identical or have mutual low-order resonances, then the oscillators can communicate provided that there are synaptic connections between them. Communication between the oscillators occurs via phases; that is, a postsynaptic oscillator changes its phase to reflect changes of phase of the presynaptic oscillator, as we illustrate in Figure 5.2.

When two oscillators have mutually nonresonant (or high-order resonant) frequency vectors, and connections between them are axo-dendritic as in Figure 4.1(c), then no communication between them is possible on the large time scale of order $1 / \varepsilon$. That is, the phase of the postsynaptic oscillator is not sensitive to that of the presynaptic one, as we illustrate in Figure 5.2. This result is universal in the sense that it does not depend on the particulars of the equations that describe dynamics of the network.

Since the frequency of quasi-periodic oscillations controls communication between oscillators, we refer to such communication as being FM (FM interaction), in analogy with FM radio. Thus, the frequency encodes the channel (or a set of channels) of communication while the information is transmitted via FMs, which are the same as PMs or phase deviations. FM interactions are employed by many biological systems, e.g., by the electrosensory system of electric fish, such as Eigenmannia [15], or by the sonar organ of echolocating bats, such as Eptesicus fuscus [39].

FM interactions can be interpreted in terms of spiking neurons as follows: The frequency of a quasi-periodically spiking cell does not carry any information other than identifying the channel of communication. The signal (neural code) is carried via timings of spikes [1].

Understanding principles of FM interactions may shed some light on why the brain exhibits rhythmic activity and why there are so many frequencies. We hypothesize that neurons or cortical columns need rhythmic activity to communicate selectively. 

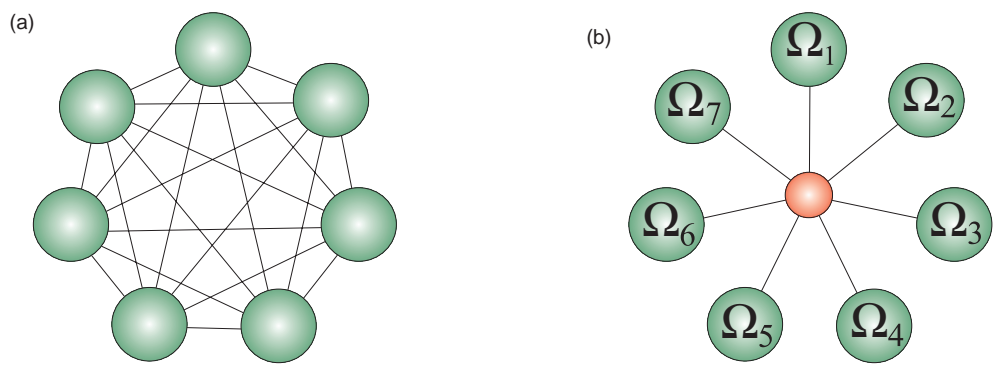

FIG. 6.1. (a) A conventional neurocomputer having $n$ neurons (shaded spheres) would have at least $n(n-1) / 2$ connections. (b) An oscillatory neurocomputer can have only $n$ connections: from each neuron to a common media (middle sphere). The neurons can communicate selectively by changing the frequency, $\Omega_{i}$, of oscillation (from [20]).

That is, cortical oscillators communicate only with those oscillators that have appropriate frequencies. They do not communicate with the other oscillators even though there might be synaptic connections between them. Thus, various ensembles of oscillators can process information without any cross interference. A cortical oscillator may participate in different ensembles simply by changing its frequency. An interesting but open question is how many different channels (ensembles) a brain can use.

We stress that we still have no idea what kind of processing of information takes place between oscillators having equal or low-order resonant frequencies, but we know that any communication is impossible between oscillators having mutually nonresonant frequencies.

6.2. Thalamo-cortical system. As we discuss in section 4.2 , an external rhythmic signal can dynamically link any two oscillators, even those that oscillate with mutually nonresonant frequencies and would have been unlinked otherwise. In terms of the thalamo-cortical system this would mean that changing its pattern of rhythmic activity, the thalamus may have complete control over the information processing taking place in the cortex.

6.3. Multiplexing. FM interactions provide a powerful mechanism for multiplexing of neural signals. Similar to the case of radio stations using the same airspace for different channels, neurons or cortical columns can use the same neural fibers to transmit on different frequencies. This mechanism does not substitute but complements the mechanism of selective communication based on anatomical selectivity of synaptic connections.

6.4. Oscillatory neurocomputers. FM interactions might prove to be useful in the design of neurocomputers, since they can avoid the $n^{2}$-connectivity problem. Indeed, a conventional neurocomputer having $n$ neurons must have at least $n(n-1) / 2$ connections (Figure 6.1(a)), which makes building such a computer for large $n$ impractical. Now suppose that each neuron is a high-frequency oscillator and the neurons communicate through a common medium (Figure 6.1(b)) so that there are only $n$ connections. Then any two neurons can change dynamically the connection between them by changing their frequencies. In particular, they can turn the connections on and off. What we have proven here and in [19] is that this mechanism would work regardless of the technical details of how the neurons are connected, what their design is, etc. A potential problem is that whenever a neuron changes its frequency, it 
alters its connections with many other neurons too. It is not clear yet how to cope with this effect or to take advantage of it. In any case, programming such an oscillatory neurocomputer would require new concepts going beyond the Hopfield network paradigm.

6.5. Dynamic link architecture. Let us contrast our results to the dynamic link architecture theory $[27,41,43]$. The latter postulates that there is a dynamic change of efficacy of synaptic connections. One of the mechanisms may be a shortterm synaptic plasticity. Another mechanism considers oscillators having identical frequencies but different phases. It postulates that in order to communicate, oscillators have to be synchronized in phase. Thus, the phase of oscillation encodes the channel of communication, while the frequency is an unused parameter.

Notice that our results and dynamic link architecture theory suggest that the entire brain could be partitioned into noninteracting ensembles processing information without any cross interference. An oscillator can change its membership in an ensemble by adjusting its frequency (in the former) or its phase (in the latter). Both theories suggest a mechanism for multiplexing of neural signals: via the frequency (in the former) or via the timing (i.e., the phase, in the latter). The theories do not contradict, but rather they complement each other. The former arises when oscillators are weakly connected, whereas the latter may be more suitable for strong connections.

6.6. Limitations. Even though we have proven that FM interactions take place in any brain model consisting of weakly connected quasi-periodic oscillators, it does not necessarily follow that FM interactions take place in the real brain since it is not clear whether or not our two major assumptions can be satisfied simultaneously in vivo.

Weak connections. Although individual cortical cells are weakly connected, the cortical columns consisting of these cells might not be as we discuss in section 1.1. The strength of connections, $\varepsilon$, between the columns is rather a variable that depends on the current state of the brain. When rhythmic activity of cortical columns results from synchronous firing of many cells, as happens in the visual cortex [13, 14], the columns are connected strongly. In contrast, if cortical oscillations involve activity of few cells with low firing rates, as it happens in areas V1, MT [4, 42, 48], the columns are connected weakly.

Quasi-periodic dynamics. There is no electrophysiological evidence suggesting that rhythmic activity of cortical columns measured via local field potentials (LFP) or EEG is close to being quasi periodic. If the rhythmic activity is chaotic or noisy, then our theory might not be applicable. It could happen, however, that the rhythmic activity is nearly quasi periodic but cannot manifest itself as such in EEG and LFP recordings due to the following reasons:

- Nearby cortical columns have quite different frequency vectors. Their overlapping quasi-periodic activity is reflected in EEG and LFP recordings having many peaks, which could be blurred by weak noise.

- Quasi-periodic activity of each cortical column becomes chaotic when the columns start to interact with other columns.

- The frequency vectors of cortical columns are not constants but slowly changing variables. The columns change their frequencies in order to participate in different dynamical ensembles and process different kinds of information. Therefore, activity of each column may consist of many short quasi-periodic episodes of duration less than $100 \mathrm{~ms}$. 
Any of these factors would lead to nondiscrete power spectra. Nevertheless, our theory would still provide a correct intuition into behavior of such a neural system.

Appendix A. Canonical models. We define canonical models according to Hoppensteadt and Izhikevich [19]. Consider a dynamical system of the form

$$
\dot{X}=F(X), \quad X \in \mathbb{R}^{m} .
$$

We say that a system

$$
\dot{x}=f(x), \quad x \in \mathbb{R}^{k},
$$

is a model of (A.1) if there is a continuous mapping $p: \mathbb{R}^{m} \rightarrow \mathbb{R}^{k}$ such that if $X(t), t \geq$ 0 , is a solution of (A.1), then $x(t)=p(X(t)), t \geq 0$, is a solution of (A.2). Thus, $p$ projects solutions of (A.1) to those of (A.2). Obviously, if $p$ is a homeomorphism, then (A.1) and (A.2) are topologically equivalent. The most interesting cases are when $k<m$, that is, when $p$ is many-to-one. We say that (A.2) is a local model of (A.1) if $p$ is defined only on an open subset $W \subset \mathbb{R}^{m}$.

Now suppose we are given a family $\mathcal{F}$ of dynamical systems of the form (A.1). We say that (A.2) is a (local) canonical model for $\mathcal{F}$ if it is a (local) model for every member of the family. Since we have little biophysical information about brain dynamics, we cannot write down a complete set of equations describing the brain, and we are forced to consider a broad family $\mathcal{F}$ of brain models. A reasonable way to study such a family is to find a canonical model for it; see a detailed discussion in $[19]$.

Appendix B. Derivation of the phase model. The representation

$$
X(t)=q(\Omega t)
$$

of a quasi-periodic signal $X(t)$ is not unique even if we demand that the frequency vector $\Omega$ be nonresonant. For example, if $A \in \mathrm{GL}(k, \mathbb{Z})$ (that is, $A$ has integer elements, it is invertible, and $A^{-1}$ has integer elements too), then $\tilde{q}=q \circ A$ and $\tilde{\Omega}=A^{-1} \Omega$ can also be used to represent $X(t)$. We say that the frequency vector $\Omega \in \mathbb{R}^{k}$ is maximal if any other frequency vector, $\tilde{\Omega} \in \mathbb{R}^{k}$, is related to $\Omega$ via the relation

$$
\Omega=A \tilde{\Omega},
$$

where $A$ is an integer matrix of rank $k$. The following lemma is needed for the proof of Theorem 2.1 .

Lemma B.1 (Samoilenko [37]). Consider a dynamical system of the form

$$
\dot{X}=F(X), \quad X \in \mathbb{R}^{m},
$$

having a quasi-periodic attractor $M$ with the dimension $k>1$. Let $\Omega \in \mathbb{R}^{k}$ be the maximal frequency vector. Then (B.1) is conjugate to

$$
\dot{\theta}=\Omega, \quad \theta \in \mathbb{T}^{k},
$$

on the attractor. That is, there is a homeomorphism $q: \mathbb{T}^{k} \rightarrow M$ such that $X(t)=$ $q(\theta(t))$ whenever $X(0) \in M$. 
In this paper we implicitly assume that all manifolds and functions are as smooth as necessary for our manipulations. In particular, we assume that the homeomorphism $q$ above is a diffeomorphism (differentiable with differentiable inverse).

Lemma B.2. An uncoupled $(\varepsilon=0)$ dynamical system of the form (2.1) has a normally hyperbolic stable compact invariant manifold $M$ (see definitions in Appendix $D)$, which is the direct product of all $M_{i}$.

This lemma is a generalization of Lemma 4.5 from [19] where a similar result was proven for the case when each $M_{i}$ is a hyperbolic limit cycle attractor. There we discuss why this result is not necessarily valid for the direct products of arbitrary normally hyperbolic smooth compact invariant manifolds.

Proof. Obviously, $M$ is compact, invariant, and stable. Let us prove its normal hyperbolicity; see the definitions in Appendix D. Since $M=M_{1} \times \cdots \times M_{n}$, we have $T M=T M_{1} \times \cdots \times T M_{n}$ and $N M=N M_{1} \times \cdots \times N M_{n}$. Therefore, $v \in T M$ can be represented as $v=v_{1}+\cdots+v_{n}$, where $v_{i} \in T M_{i}$. Similarly, $w=w_{1}+\cdots+w_{n}$ for $w_{i} \in N M_{i}, i=1, \ldots, n$. From exponential stability of each $M_{i}$ it follows that $\lim _{t \rightarrow \infty}\left|w_{i}(t)\right|=0$, and hence $\lim _{t \rightarrow \infty}|w(t)|=0$ (which implies exponential stability of $M)$.

To determine dynamics of $v_{i}(t)$ we need to find the flow $\Phi_{i}\left(X_{i}, t\right)$ on $M_{i}$. Using Lemma B.1 we see that $\Phi_{i}\left(X_{i}, t\right)=q_{i}\left(\Omega_{i} t+q_{i}^{-1}\left(X_{i}\right)\right)$ for some maximal frequency vector $\Omega_{i}$ and some diffeomorphism $q_{i}: \mathbb{T}^{k} \rightarrow M_{i}$. Therefore

$$
D_{X_{i}} \Phi_{i}\left(X_{i}, t\right)=D q_{i}\left(\Omega_{i} t+q_{i}^{-1}\left(X_{i}\right)\right) D q_{i}^{-1}\left(X_{i}\right)=D q_{i}\left(\Omega_{i} t+\theta_{i}\right) D q_{i}\left(\theta_{i}\right)^{-1},
$$

where $\theta_{i}=q_{i}^{-1}\left(X_{i}\right)$. Therefore,

$$
\begin{aligned}
\left|v_{i}(t)\right| & =\left|D_{X_{i}} \Phi_{i}\left(X_{i}, t\right) v_{i}(0)\right| \\
& =\left|D q_{i}\left(\Omega_{i} t+\theta_{i}\right) D q_{i}\left(\theta_{i}\right)^{-1} v_{i}(0)\right| \\
& \geq \min _{\vartheta_{i}, \theta_{i} \in \mathbb{T}^{k}}\left|D q_{i}\left(\vartheta_{i}\right) D q_{i}\left(\theta_{i}\right)^{-1} v_{i}(0)\right| \\
& \geq\left|\lambda_{\min }\left(D q_{i}\right)\right| /\left|\lambda_{\max }\left(D q_{i}\right)\right|\left|v_{i}(0)\right|,
\end{aligned}
$$

where $\lambda_{\min }\left(D q_{i}\right)$ and $\lambda_{\max }\left(D q_{i}\right)$ are the minimal and the maximal (in absolute value) eigenvalues of $D q_{i}$ on $\mathbb{T}^{k}$, respectively. They exist since $\mathbb{T}^{k}$ is compact. Moreover, $\lambda_{\min }\left(D q_{i}\right) \neq 0$, since $q_{i}$ is nonsingular on $\mathbb{T}^{k}$. It follows that each $\left|v_{i}(t)\right|$ is uniformly bounded from 0 , and hence $|v(t)|$ is too. Therefore, $\lim _{t \rightarrow \infty}|w(t)| /|v(t)|=0$, which completes the proof.

Now we are ready to prove Theorem 2.1. First, we apply Lemma B.2 to conclude that the system (2.1) for $\varepsilon=0$ has a normally hyperbolic stable compact invariant manifold $M$. Then, we use Theorem D.1 to determine the existence of an open neighborhood $W$ of $M$ and a mapping $p_{W}: W \rightarrow M$ that projects all local solutions of (2.1) to those of the system

$$
\dot{x}_{i}=F_{i}\left(x_{i}\right)+\varepsilon g_{i}\left(x_{1}, \ldots, x_{n}, \varepsilon\right), \quad x_{i} \in M_{i}, \quad i=1, \ldots, n,
$$

where each $F_{i}$ is the same as in (2.1), and $g_{i}$ are some functions.

Notice that the inverse of $q_{i}$, which we denote by $p_{i}=q_{i}^{-1}: M_{i} \rightarrow \mathbb{T}^{k}$, transforms each subsystem $\dot{x}_{i}=F_{i}\left(x_{i}\right)$ into the form $\dot{\theta}_{i}=\Omega_{i}$; see Lemma B.1. Differentiating $\theta_{i}(t)=p_{i}\left(x_{i}(t)\right)$ with respect to $t$ yields

$$
\Omega_{i}=D p_{i}\left(x_{i}\right) F_{i}\left(x_{i}\right)
$$


for all $x_{i} \in M_{i}$. Now we apply the diffeomorphism to the weakly connected system (B.2) to obtain

$$
\dot{\theta}_{i}=D p_{i}\left(x_{i}\right)\left[F_{i}\left(x_{i}\right)+\varepsilon g_{i}\left(x_{1}, \ldots, x_{n}, \varepsilon\right)\right]=\Omega_{i}+\varepsilon h_{i}\left(\theta_{1}, \ldots, \theta_{n}, \varepsilon\right),
$$

where

$$
h_{i}\left(\theta_{1}, \ldots, \theta_{n}, \varepsilon\right)=D p_{i}\left(q_{i}\left(\theta_{i}\right)\right) g_{i}\left(q_{1}\left(\theta_{1}\right), \ldots, q_{n}\left(\theta_{n}\right), \varepsilon\right) .
$$

The mapping $p$ that transforms solutions of (2.1) to those of (2.3) is the superposition of $p_{W}: W \rightarrow M$ and $\left(p_{1}, \ldots, p_{n}\right): M \rightarrow \mathbb{T}^{n k}$.

Appendix C. Proof of Theorem 3.1. Let $\phi(t)=\theta(t)-\Omega t$ be the vector of phase deviations. Then

$$
\dot{\phi}=\varepsilon h(\Omega t+\phi, \varepsilon) .
$$

Let

$$
Q(\phi)=\lim _{T \rightarrow \infty} \frac{1}{T} \int_{0}^{T} h(\Omega t+\phi, 0) d t
$$

denote the average of $h$. Then the nearly identity change of variables, which is equivalent to formal averaging

$$
\phi=\varphi+\varepsilon \int_{0}^{t} h(\Omega s+\varphi, 0)-Q(\varphi) d s
$$

transforms (C.1) into the "averaged" system

$$
\dot{\varphi}=\varepsilon Q(\varphi)+o(\varepsilon) .
$$

From Lemma 9.5 in [19] it follows that there is an $\varepsilon_{0}>0$ such that $|\phi(t)-\varphi(t)|=o(1)$ uniformly for all $\varepsilon \leq \varepsilon_{0}$ and all $t$ on a time scale of order $1 / \varepsilon$.

Now we use the theorem below to determine the constant $\omega$, or the function $H$ such that $Q(\varphi)=H(K \varphi)$, where $K$ is the resonant matrix for $\Omega$. Finally, we define

$$
\vartheta(t)=\Omega t+\bar{\varphi}(t) .
$$

It is easy to check that $\vartheta$ is governed by (3.3) or (3.4) depending on the existence of resonances in $\Omega$.

Theorem C.1 (Hoppensteadt and Izhikevich [19]). Let $h$ be a function defined on $\mathbb{T}^{n}$ having absolutely convergent Fourier series. Let $Q$ be defined by

$$
Q(\varphi)=\lim _{T \rightarrow \infty} \frac{1}{T} \int_{0}^{T} h(\Omega t+\varphi) d t
$$

where $\Omega \in \mathbb{R}^{n}$ is a vector of frequencies.

- If $\Omega$ is nonresonant (that is, $k \Omega \neq 0$ for all nonzero $k \in \mathbb{Z}^{n}$ ), then $Q(\varphi)=\omega$, where $\omega$ is the constant term in the Fourier series of $h$.

- If $\Omega$ is resonant and $K$ is the corresponding resonant matrix, then there is a smooth function $H$ defined on a lower-dimensional torus $\mathbb{T}^{s}=K \mathbb{T}^{n}$ such that $Q(\varphi)=H(K \varphi)$. 


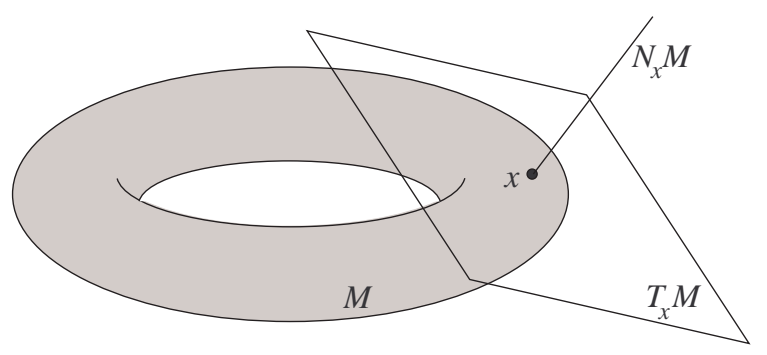

FiG. D.1. Tangent $T_{x} M$ and normal $N_{x} M$ linear subspaces to manifold $M$ (from [19]).

Recall that a sufficient condition for an absolute convergence of Fourier series of $h$ is its continuous differentiability, which is more than enough for our applications.

Appendix D. Normally hyperbolic invariant manifolds. We define normally hyperbolic invariant manifolds following [11, 44, 19]. We consider

$$
\dot{X}=F(X), \quad X \in \mathbb{R}^{m},
$$

in a neighborhood of a compact invariant manifold $M$. There are two linear mutually orthogonal spaces associated with each point $X \in M$ : the tangent space, denoted by $T_{X} M$, and the normal space, denoted by $N_{X} M$ [17]. The tangent space can be thought of as the collection of all lines tangent to $M$ at $X$; see the illustration in Figure D.1.

Let

$$
\Pi: T_{X} M \rightarrow N_{X} M
$$

be the orthogonal projection to the normal subspace $N_{X} M$. To define the notion of contractions of vectors at $M$, we consider the linear part, $D_{X} \Phi(X, t)$, of the flow $\Phi(X, t)$ at the invariant manifold $M$. Let

$$
\begin{array}{ll}
v(t)=D_{X} \Phi(X, t) v(0), & v(0) \in T_{X} M, \\
w(t)=\Pi D_{X} \Phi(X, t) w(0), & w(0) \in N_{X} M .
\end{array}
$$

The invariant manifold is normally hyperbolic when

$$
\lim _{t \rightarrow \infty}|w(t)|=0 \quad \text { (exponential stability) }
$$

and

$$
\lim _{t \rightarrow \infty} \frac{|w(t)|}{|v(t)|}=0 \quad \text { (normal hyperbolicity) }
$$

for all $X \in M$ and all nonzero vectors $w \in N_{X} M$ and $v \in T_{X} M$. The latter condition means that the rate of normal contraction to the manifold is larger than the tangential one; see the illustration in Figure D.2.

Normally hyperbolic smooth compact invariant manifolds possess a useful property: they are persistent under perturbations $[11,16]$. This implies the following result. 


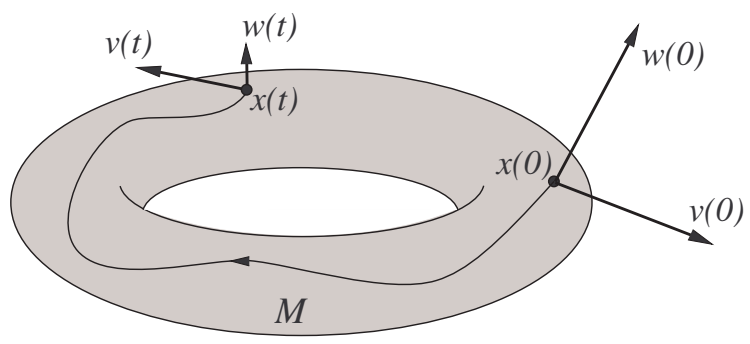

FiG. D.2. Normal contraction to the manifold is sharper than the tangential one (from [19]).

Theorem D.1 (Hoppensteadt and Izhikevich [19]). Suppose a dynamical system

$$
\dot{X}=F(X), \quad X \in \mathbb{R}^{m},
$$

has an attractive normally hyperbolic compact invariant manifold $M \subset \mathbb{R}^{m}$. Then there is an $\varepsilon_{0}>0$ such that for all $\varepsilon \leq \varepsilon_{0}$ the dynamical system

$$
\dot{X}=F(X)+\varepsilon G(X, \varepsilon), \quad X \in \mathbb{R}^{m},
$$

has a local model, which is defined on the unperturbed invariant manifold $M$ :

$$
\dot{x}=F(x)+\varepsilon g(x, \varepsilon), \quad x \in M,
$$

where

$$
g(x, 0)=G(x, 0)+\operatorname{ad}_{F} P(x), \text { where } \operatorname{ad}_{F} P=D F P-D P F
$$

is the Poisson bracket of the vector field $F$ and the vector-valued function $P$ that can be determined from the condition

$$
G(x, 0)+\operatorname{ad}_{F} P(x) \in T_{x} M \quad \text { for all } x \in M .
$$

That is, there is an open neighborhood $W$ of $M$, and a continuous transformation $p_{W}: W \rightarrow M$ that maps solutions of (D.1) to those of (D.2).

It is a hard mathematical problem to find $P$ in applications. The Malkin theorem [19] covers some special cases.

Acknowledgments. The author thanks Frank Hoppensteadt who inspired the writing of this paper. J. Guckenheimer, M. Michael Boyle, Morris W. Hirsch, and Marek Rychlik helped to clarify a few issues. Their assistance is highly appreciated. Special thanks to Arthur Sherman who made an excellent choice of peer reviewers whose criticism and suggestions helped to improve the quality of the manuscript. John M. Burke Jr. helped to improve the readability of the manuscript.

\section{REFERENCES}

[1] M. Abeles, Y. Prut, H. Bergman, and E. Vaadia, Synchronization in neuronal transmission and its importance for information processing, in Temporal Coding in the Brain, G. Buzsáki, R. Llinás, W. Singer, A. Berthoz, and Y. Christen eds., Springer-Verlag, New York 1994. 
[2] M. A. Arbib, P. Érdi, And J. Szentágothai, Neural Organization, Mit Press, Cambridge, MA, 1998.

[3] C. Baesens, J. Guckenheimer, S. Kim, and R. S. Mackay, Three coupled oscillators: Modelocking, global bifurcations and toroidal chaos, Phys. D, 49 (1991), pp. 387-475.

[4] W. Bair, C. Koch, W. Newsome, K. Britten, And E. Niebur, Power spectrum analysis of bursting cells in area MT in the behaving monkey, J. Neurosci., 14 (1994), pp. 2870-2892.

[5] N. Yu. Bibikov, Multi-Frequency Nonlinear Oscillations and Their Bifurcations, Leningrad University Press, Leningrad, 1991 (in Russian).

[6] G. N. Borisyuk, R. M. Borisyuk, A. I. Khibnik, And D. Roose, Dynamics and bifurcations of two coupled neural oscillators with different connection types, Bull. Math. Biol., 57 (1995), pp. 809-840.

[7] H. W. Broer, G. B. Huitema, and M. B. Sevryuk, Quasi-Periodic Motions in Families of Dynamical Systems, Springer-Verlag, Berlin, Heidelberg, 1996.

[8] G. Buzsáki, Z. Horváth, R. Urioste, J. Hetke, and K. Wise, High-frequency network oscillation in the hippocampus, Science, 256 (1992), pp. 1025-1027.

[9] G. B. Ermentrout, n:m phase-locking of weakly coupled oscillators, J. Math. Biol., 12 (1981), pp. $327-342$.

[10] G. B. ERmentrout And N. Kopell, Multiple pulse interactions and averaging in systems of coupled neural oscillators, J. Math. Biol., 29 (1991), pp. 195-217.

[11] N. Fenichel, Persistence and smoothness of invariant manifolds for flows, Indiana Univ. Math. J., 21 (1971/1972), pp. 193-226.

[12] Y. Frégnac, V. Bringuier, and A. Baranyi, Oscillatory neuronal activity in visual cortex: A critical re-evaluation, in Temporal Coding in the Brain, G. Buzsáki, R. Llinás, W. Singer, A. Berthoz, and Y. Christen eds., Springer-Verlag, Berlin, New York, 1994.

[13] C. M. GraY, Synchronous oscillations in neuronal systems: Mechanism and functions, J. Comput. Neurosci., 1 (1994), pp. 11-38.

[14] C. M. Gray, P. Konig, A. K. Engel, And W. Singer, Oscillatory responses in cat visual cortex exhibit inter-columnar synchronization which reflects global stimulus properties, Nature, 338 (1989), pp. 334-337.

[15] W. Heiligenberg, The coding and processing of temporal information in the electrosensory system of fish, in Temporal Coding in the Brain, G. Buzsáki, R. Llinás, W. Singer, A. Berthoz, and Y. Christen, eds., Springer-Verlag, Berlin, New York, 1994.

[16] M. W. Hinsch, C. C. Pugh, And M. Shub, Invariant Manifolds, Springer-Verlag, New York, 1977.

[17] M. W. Hinsch, Differential Topology, Springer-Verlag, New York, 1976.

[18] F. C. Hoppensteadt and E. M. Izhikevich, Synaptic Organizations and Dynamical Properties of Weakly Connected Neural Oscillators: I. Analysis of Canonical Model, Biological Cybernetics, 75 (1996), pp. 117-127.

[19] F. C. Hoppensteadt and E. M. Izhikevich, Weakly Connected Neural Networks, SpringerVerlag, New York, 1997.

[20] E. M. IzHIKeVICH, Weakly pulse-coupled oscillators, FM interactions, synchronization, and oscillatory associative memory, IEEE Trans. Neural Networks, 10 (1999), pp. 508-526.

[21] Ya.B. KazAnOvich AND R. M. BorisyUk, Synchronization in a neural network of phase oscillators with the central element, Biological Cybernetics, 71 (1994), pp. 177-185.

[22] N. Kopell, Chains of coupled oscillators, in Brain Theory and Neural Networks, M.A. Arbib, ed., MIT Press, Cambridge, MA, 1995, pp. 178-183.

[23] Y. Kuramoto, Collective synchronization of pulse-coupled oscillators and excitable units, Phys. D, 50 (1991), pp. 15-30.

[24] Y. Kuramoto, Chemical Oscillations, Waves, and Turbulence, Springer-Verlag, New York, 1984.

[25] R. R. LLINÁs, The intrinsic electrophysiological properties of mammalian neurons: A new insight into CNS function, Science, 242 (1988), pp. 1654-1664.

[26] R. R. LlinÁs, A. A. Grace, and Y. Yarom, In vitro neurons in mammalian cortical layer 4 exhibit intrinsic oscillatory activity in the 10- to 50-Hz frequency range, Proc. Natl. Acad. Sci., 88 (1991), pp. 897-901.

[27] C. von der Malsburg, Dynamic link architecture, in Brain Theory and Neural Networks, M.A. Arbib, ed., MIT Press, Cambridge, MA, 1995, pp. 329-331.

[28] A. Mason, A. Nicoll, And K. Stratford, Synaptic transmission between individual pyramidal neurons of the rat visual cortex in vitro, J. Neurosci., 11 (1991), pp. 72-84.

[29] B. L. Mcnaughton, C. A. Barnes, And P. Andersen, Synaptic efficacy and EPSP summation in granule cells of rat fascia dentata studied in vitro, J. Neurophysiol., 46 (1981), pp. 952-966. 
[30] R. Miles ANd R. K. S. Wong, Excitatory synaptic interactions between CA3 neurons in the guinea-pig hippocampus, J. Physiol., 373 (1986), pp. 397-418.

[31] R. E. Mirollo And S. H. Strogatz, Synchronization of pulse-coupled biological oscillators, SIAM J. Appl. Math., 50 (1990), pp. 1645-1662.

[32] S. E. Newhouse, D. Ruelle, and F. Takens, Occurrence of strange axiom-A attractors near quasi-periodic flows on $\mathbb{T}^{m}, m \geq 3$, Comm. Math. Phys., 64 (1978), pp. 35-40.

[33] P. L. Nunez, Neocortical Dynamics and Human EEG Rhythms, Oxford University Press, New York, London, 1995.

[34] C. S. Peskin, Mathematical Aspects of Heart Physiology, Courant Institute of Mathematical Sciences, New York University, New York, 1975.

[35] R. H. Rand, A. H. Cohen, And P. J. Holmes, Systems of coupled oscillators as models of central pattern generators, in Neural Control of Rhythmic Movements in Vertebrates, A. H Cohen, S. Grillner, and S. Rossignol, eds., Wiley, New York, 1987, pp. 369-413.

[36] D. Ruelle and F. Takens, On the nature of turbulence, Comm. Math. Phys., 20 (1971), pp. $167-192$.

[37] A. M. SAmoilenko, Elements of the Mathematical Theory of Multi-Frequency Oscillations, Mathematics and Its Applications (Soviet Series) 71, Kluwer Academic Publishers, Dordrecht, the Netherlands, 1991.

[38] R. J. Sayer, M. J. Friedlander, and S. J. Redman, The time course and amplitude of EPSPs evoked at synapses between pairs of CA3/CA1 neurons in the hippocampal slice, J. Neurosci., 10 (1990), pp. 826-836.

[39] J. A. Simmons, M. Ferragamo, C. F. Moss, S. B. Stevenson, and R. A. Altes, Discrimination of jittered sonar echoes by the echolocating bat, Eptesicus fuscus: The shape of target images in echolocation, J. Comp. Physiol., A167 (1990), pp. 589-616.

[40] W. Singer And C. M. Gray, Visual feature integration and the temporal correlation hypothesis, Ann. Rev. Neurosci., 18 (1995), pp. 555-586.

[41] D. Terman And D. L. WANG, Global competition and local cooperation in a network of neural oscillators, Phys. D, 81 (1995), pp. 148-176.

[42] M. J. Tovee AND E. T. Rolls, Oscillatory activity is not evident in the primate temporal visual cortex with static stimuli, NeuroReport, 3 (1992), pp. 369-372.

[43] D. L. WANG, Emergent synchrony in locally coupled neural oscillators, IEEE Trans. Neural Networks, 6 (1995), pp. 941-948.

[44] S. Wiggins, Normally Hyperbolic Invariant Manifolds in Dynamical Systems, Springer-Verlag, New York, 1994.

[45] H. R. Wilson AND J. D. Cowan, Excitatory and inhibitory interaction in localized populations of model neurons, Biophys. J., 12 (1972), pp. 1-24.

[46] H. R. Wilson AND J. D. CowAn, A mathematical theory of the functional dynamics of cortical and thalamic nervous tissue, Kybernetik, 13 (1973), pp. 55-80.

[47] A. Winfree, The Geometry of Biological Time, Springer-Verlag, New York, 1980.

[48] M. P Young, K. Tanaka, and S. Yamane, On oscillating neuronal responses in the visual cortex of the monkey, J. Neurophysiol., 67 (1992), pp. 1464-1474. 